EUROPEAN ORGANISATION FOR NUCLEAR RESEARCH

CERN-PPE/95-06

20th January 95

\title{
Measurement of the Leptonic Branching Ratios of the $\tau$ lepton
}

\section{The OPAL Collaboration}

\begin{abstract}
The leptonic branching ratios of the tau lepton have been determined from data collected by the OPAL detector in 1991 and 1992. From a sample of $27196 \mathrm{e}^{+} \mathrm{e}^{-} \rightarrow \tau^{+} \tau^{-}$candidates we find $7322 \tau \rightarrow \mathrm{e} \nu \bar{\nu}$ and $7941 \tau \rightarrow \mu \nu \bar{\nu}$ candidates. Using efficiency and background estimates determined from a study of Monte Carlo events and control samples of data, the branching ratios $B(\tau \rightarrow \mathrm{e} \nu \bar{\nu})=(18.14 \pm 0.20 \pm 0.28) \%$ and $B(\tau \rightarrow \mu \nu \bar{\nu})=(17.48 \pm 0.18 \pm 0.23) \%$ have been obtained. These new results have been combined with the published results for the 1990 OPAL data to yield the following branching ratios for data taken between 1990 and 1992:

$$
\begin{aligned}
& B(\tau \rightarrow \mathrm{e} \nu \bar{\nu})=(18.04 \pm 0.33) \% \\
& B(\tau \rightarrow \mu \nu \bar{\nu})=(17.36 \pm 0.27) \%
\end{aligned}
$$

These leptonic branching ratios are used with other properties of the muon and tau-lepton to test the universality of charged current leptonic couplings in these decays.

The ratio $R_{\tau}=B\left(\tau \rightarrow\right.$ hadrons $\left.+\nu_{\tau}\right) / B\left(\tau \rightarrow \mathrm{e} \bar{\nu}_{\mathrm{e}} \nu_{\tau}\right)$ is calculated using our measured values of the leptonic branching fractions of the tau and tau lifetime from which a value of $\alpha_{s}\left(Q^{2}=M_{\tau}^{2}\right)$ is extracted. The value of $\alpha_{s}\left(Q^{2}=M_{Z}^{2}\right)$ is obtained by $Q^{2}$ evolution and agrees with the value from the $Z^{0}$ line shape analysis.
\end{abstract}

(to be submitted to Zeitschrift für Physik C) 


\section{The OPAL Collaboration}

R. Akers ${ }^{16}$, G. Alexander ${ }^{23}$, J. Allison ${ }^{16}$, K. Ametewee ${ }^{25}$, K.J. Anderson ${ }^{9}$, S. Arcelli ${ }^{2}$, S. Asai ${ }^{24}$, D. Axen ${ }^{29}$, G. Azuelos ${ }^{18, a}$, A.H. Ball ${ }^{17}$, E. Barberio ${ }^{26}$, R.J. Barlow ${ }^{16}$, R. Bartoldus ${ }^{3}$, J.R. Batley ${ }^{5}$, G. Beaudoin ${ }^{18}$, A. Beck ${ }^{23}$, G.A. Beck ${ }^{13}$, C. Beeston ${ }^{16}$, T. Behnke ${ }^{27}$, K.W. Bell ${ }^{20}$, G. Bella ${ }^{23}$, S. Bentvelsen ${ }^{8}$, P. Berlich ${ }^{10}$, S. Bethke ${ }^{32}$, O. Biebel ${ }^{32}$, I.J. Bloodworth ${ }^{1}$, P. Bock ${ }^{11}$, H.M. Bosch ${ }^{11}$, M. Boutemeur ${ }^{18}$, S. Braibant ${ }^{12}$, P. Bright-Thomas ${ }^{25}$, R.M. Brown ${ }^{20}$, A. Buijs ${ }^{8}$, H.J. Burckhart ${ }^{8}$, R. Bürgin ${ }^{10}$, C. Burgard ${ }^{27}$, N. Capdevielle ${ }^{18}$, P. Capiluppi ${ }^{2}$, R.K. Carnegie ${ }^{6}$,

A.A. Carter $^{13}$, J.R. Carter ${ }^{5}$, C.Y. Chang ${ }^{17}$, C. Charlesworth ${ }^{6}$, D.G. Charlton ${ }^{1, b}$, S.L. Chu ${ }^{4}$,

P.E.L. Clarke ${ }^{15}$, J.C.Clayton ${ }^{1}$, S.G. Clowes $^{16}$, I. Cohen ${ }^{23}$, J.E. Conboy ${ }^{15}$, O.C. Cooke ${ }^{16}$, M. Cuffiani ${ }^{2}$, S. Dado ${ }^{22}$, C. Dallapiccola ${ }^{17}$, G.M. Dallavalle ${ }^{2}$, C. Darling ${ }^{31}$, S. De Jong ${ }^{12}$, L.A. del

$\mathrm{Pozo}^{8}$, H. Deng ${ }^{17}$, M. Dittmar ${ }^{4}$, M.S. Dixit ${ }^{7}$, E. do Couto e Silva ${ }^{12}$, J.E. Duboscq ${ }^{8}$,

E. Duchovni ${ }^{26}$, G. Duckeck ${ }^{8}$, I.P. Duerdoth ${ }^{16}$, U.C. Dunwoody ${ }^{5}$, J.E.G. Edwards ${ }^{16}$,

P.A.Elcombe ${ }^{5}$, P.G. Estabrooks ${ }^{6}$, E. Etzion ${ }^{23}$, H.G. Evans ${ }^{9}$, F. Fabbri ${ }^{2}$, B. Fabbro ${ }^{21}$, M. Fanti ${ }^{2}$, P. Fath ${ }^{11}$, M. Fierro ${ }^{2}$, M. Fincke-Keeler ${ }^{28}$, H.M. Fischer ${ }^{3}$, P. Fischer ${ }^{3}$, R. Folman ${ }^{26}$, D.G. Fong ${ }^{17}$,

M. Foucher ${ }^{17}$, H. Fukui ${ }^{24}$, A. Fürtjes ${ }^{8}$, P. Gagnon ${ }^{6}$, A. Gaidot ${ }^{21}$, J.W. Gary ${ }^{4}$, J. Gascon ${ }^{18}$,

N.I. Geddes ${ }^{20}$, C. Geich-Gimbel ${ }^{3}$, S.W. Gensler ${ }^{9}$, F.X. Gentit ${ }^{21}$, T. Geralis ${ }^{20}$, G. Giacomelli ${ }^{2}$,

P. Giacomelli ${ }^{4}$, R. Giacomelli ${ }^{2}$, V. Gibson ${ }^{5}$, W.R. Gibson ${ }^{13}$, J.D. Gillies ${ }^{20}$, J. Goldberg ${ }^{22}$,

D.M. Gingrich ${ }^{30, a}$, M.J. Goodrick ${ }^{5}$, W. Gorn ${ }^{4}$, C. Grandi ${ }^{2}$, E. Gross ${ }^{26}$, J. Hagemann ${ }^{27}$, G.G. Hanson ${ }^{12}$, M. Hansroul ${ }^{8}$, C.K. Hargrove ${ }^{7}$, P.A. Hart ${ }^{9}$, M. Hauschild ${ }^{8}$, C.M. Hawkes ${ }^{8}$, E. Heflin ${ }^{4}$, R.J. Hemingway ${ }^{6}$, G. Herten ${ }^{10}$, R.D. Heuer ${ }^{8}$, J.C.Hill ${ }^{5}$, S.J. Hillier ${ }^{8}$, T. Hilse ${ }^{10}$, P.R.Hobson ${ }^{25}$, D. Hochman ${ }^{26}$, R.J. Homer ${ }^{1}$, A.K. Honma ${ }^{28, a}$, R. Howard ${ }^{29}$,

R.E. Hughes-Jones ${ }^{16}$, P. Igo-Kemenes ${ }^{11}$, D.C. Imrie $^{25}$, A. Jawahery ${ }^{17}$, P.W. Jeffreys ${ }^{20}$, H. Jeremie ${ }^{18}$, M. Jimack ${ }^{1}$, M. Jones ${ }^{6}$, R.W.L. Jones ${ }^{8}$, P. Jovanovic ${ }^{1}$, C. Jui ${ }^{4}$, D. Karlen ${ }^{6}$, J. Kanzaki ${ }^{24}$, K. Kawagoe ${ }^{24}$, T. Kawamoto ${ }^{24}$, R.K. Keeler ${ }^{28}$, R.G. Kellogg ${ }^{17}$, B.W. Kennedy ${ }^{20}$, B. King ${ }^{8}$, J. King ${ }^{13}$, J. Kirk ${ }^{29}$, S. Kluth ${ }^{5}$, T. Kobayashi ${ }^{24}$, M. Kobel ${ }^{10}$, D.S. Koetke ${ }^{6}$, T.P. Kokott ${ }^{3}$, S. Komamiya ${ }^{24}$, R. Kowalewski ${ }^{8}$, T. Kress ${ }^{11}$, P. Krieger ${ }^{6}$, J. von Krogh ${ }^{11}$, P. Kyberd ${ }^{13}$, G.D. Lafferty ${ }^{16}$, H. Lafoux ${ }^{8}$, R. Lahmann ${ }^{17}$, W.P. Lai ${ }^{19}$, J. Lauber ${ }^{8}$, J.G. Layter ${ }^{4}$, P. Leblanc ${ }^{18}$, A.M. Lee ${ }^{31}$, E. Lefebvre ${ }^{18}$, D. Lellouch ${ }^{26}$, C. Leroy ${ }^{18}$, J. Letts ${ }^{2}$, L. Levinson ${ }^{26}$, S.L. Lloyd ${ }^{13}$, F.K. Loebinger ${ }^{16}$, G.D. Long ${ }^{17}$, B. Lorazo $^{18}$, M.J. Losty ${ }^{7}$, X.C. Lou ${ }^{8}$, J. Ludwig ${ }^{10}$, A. Luig ${ }^{10}$, M. Mannelli ${ }^{8}$, S. Marcellini ${ }^{2}$, C. Markus ${ }^{3}$, A.J. Martin ${ }^{13}$, J.P. Martin ${ }^{18}$, T.Mashimo ${ }^{24}$, W. Matthews ${ }^{25}$, P.Mättig ${ }^{3}$, U. Maur ${ }^{3}$, J. McKenna ${ }^{29}$, T.J.McMahon ${ }^{1}$,

A.I. McNab ${ }^{13}$, F. Meijers ${ }^{8}$, F.S. Merritt ${ }^{9}$, H. $\mathrm{Mes}^{7}$, A. Michelini ${ }^{8}$, R.P. Middleton ${ }^{20}$, G. Mikenberg ${ }^{26}$, D.J. Miller ${ }^{15}$, R. Mir $^{26}$, W. Mohr ${ }^{10}$, A. Montanari ${ }^{2}$, T. Mori ${ }^{24}$, M. Morii ${ }^{24}$, U. Müller ${ }^{3}$, B. Nellen ${ }^{3}$, B. Nijjhar ${ }^{16}$, S.W. O'Neale', F.G. Oakham ${ }^{7}$, F. Odorici ${ }^{2}$, H.O. Ogren ${ }^{12}$, N.J.Oldershaw ${ }^{16}$, C.J. Oram ${ }^{28, a}$, M.J. Oreglia ${ }^{9}$, S. Orito ${ }^{24}$, F. Palmonari ${ }^{2}$, J.P. Pansart ${ }^{21}$,

G.N.Patrick ${ }^{20}$, M.J. Pearce ${ }^{1}$, P.D. Phillips ${ }^{16}$, J.E. Pilcher ${ }^{9}$, J.Pinfold ${ }^{30}$, D.E. Plane ${ }^{8}$, P.Poffenberger ${ }^{28}$, B. Poli ${ }^{2}$, A. Posthaus ${ }^{3}$, T.W. Pritchard ${ }^{13}$, H. Przysiezniak ${ }^{30}$,

M.W. Redmond ${ }^{8}$, D.L. Rees $^{8}$, D. Rigby ${ }^{1}$, M.G. Rison ${ }^{5}$, S.A. Robins ${ }^{13}$, D. Robinson ${ }^{5}$, N. Rodning ${ }^{30}$, J.M. Roney ${ }^{28}$, E. $\operatorname{Ros}^{8}$, A.M. Rossi ${ }^{2}$, M. Rosvick ${ }^{28}$, P. Routenburg ${ }^{30}$, Y. Rozen ${ }^{8}$, K. Runge ${ }^{10}$, O. Runolfsson ${ }^{8}$, D.R. Rust ${ }^{12}$, M.Sasaki ${ }^{24}$, C.Sbarra ${ }^{2}$, A.D.Schaile ${ }^{8}$, O.Schaile ${ }^{10}$, F.Scharf ${ }^{3}$, P.Scharff-Hansen ${ }^{8}$, P. Schenk ${ }^{4}$, B.Schmitt ${ }^{3}$, M.Schröder ${ }^{8}$, H.C.Schultz-Coulon ${ }^{10}$, P.Schütz ${ }^{3}$, M.Schulz ${ }^{8}$, C.Schwick ${ }^{27}$, J.Schwiening ${ }^{3}$, W.G.Scott ${ }^{20}$, M.Settles ${ }^{12}$, T.G.Shears ${ }^{5}$,

B.C.Shen ${ }^{4}$, C.H.Shepherd-Themistocleous ${ }^{7}$, P. Sherwood ${ }^{15}$, G.P. Siroli ${ }^{2}$, A. Skillman ${ }^{15}$, A.Skuja ${ }^{17}$, A.M.Smith ${ }^{8}$, T.J.Smith ${ }^{28}$, G.A.Snow ${ }^{17}$, R. Sobie ${ }^{28}$, S. Söldner-Rembold ${ }^{10}$, R.W.Springer ${ }^{30}$, M.Sproston ${ }^{20}$, A.Stahl ${ }^{3}$, M.Starks ${ }^{12}$, C.Stegmann ${ }^{10}$, K.Stephens ${ }^{16}$, J. Steuerer ${ }^{28}$, B. Stockhausen ${ }^{3}$, D. Strom ${ }^{19}$, P. Szymanski ${ }^{20}$, R. Tafirout ${ }^{18}$, H. Takeda ${ }^{24}$, 


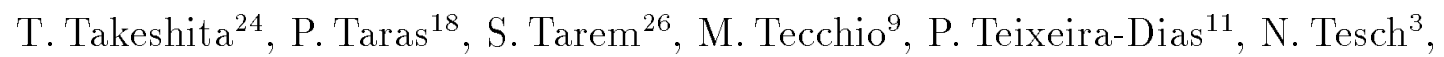
M.A. Thomson ${ }^{8}$, O. Tousignant ${ }^{18}$, S. Towers $^{6}$, M. Tscheulin ${ }^{10}$, T. Tsukamoto ${ }^{24}$, A. Turcot ${ }^{9}$, M.F. Turner-Watson ${ }^{8}$, P. Utzat ${ }^{11}$, R. Van Kooten ${ }^{12}$, G. Vasseur ${ }^{21}$, P. Vikas ${ }^{18}$, M. Vincter ${ }^{28}$,

A. Wagner ${ }^{27}$, D.L. Wagner ${ }^{9}$, C.P. Ward ${ }^{5}$, D.R. Ward ${ }^{5}$, J.J. Ward ${ }^{15}$, P.M. Watkins ${ }^{1}$, A.T. Watson ${ }^{1}$, N.K. Watson ${ }^{7}$, P. Weber ${ }^{6}$, P.S. Wells ${ }^{8}$, N. Wermes ${ }^{3}$, B. Wilkens ${ }^{10}$, G.W.Wilson ${ }^{27}$, J.A. Wilson ${ }^{1}$, V-H. Winterer ${ }^{10}$, T. Wlodek ${ }^{26}$, G. Wolf ${ }^{26}$, S. Wotton ${ }^{11}$, T.R. Wyatt ${ }^{16}$, A. Yeaman ${ }^{13}$, G. Yekutieli ${ }^{26}$, M. Yurko ${ }^{18}$, V.Zacek ${ }^{18}$, W. Zeuner ${ }^{8}$, G.T. Zorn ${ }^{17}$.

${ }^{1}$ School of Physics and Space Research, University of Birmingham, Birmingham B15 2TT, UK

${ }^{2}$ Dipartimento di Fisica dell' Università di Bologna and INFN, I-40126 Bologna, Italy

${ }^{3}$ Physikalisches Institut, Universität Bonn, D-53115 Bonn, Germany

${ }^{4}$ Department of Physics, University of California, Riverside CA 92521, USA

${ }^{5}$ Cavendish Laboratory, Cambridge CB3 0HE, UK

${ }^{6}$ Carleton University, Department of Physics, Colonel By Drive, Ottawa, Ontario K1S 5B6, Canada

${ }^{7}$ Centre for Research in Particle Physics, Carleton University, Ottawa, Ontario K1S 5B6, Canada

${ }^{8}$ CERN, European Organisation for Particle Physics, CH-1211 Geneva 23, Switzerland

${ }^{9}$ Enrico Fermi Institute and Department of Physics, University of Chicago, Chicago IL 60637 , USA

${ }^{10}$ Fakultät für Physik, Albert Ludwigs Universität, D-79104 Freiburg, Germany

${ }^{11}$ Physikalisches Institut, Universität Heidelberg, D-69120 Heidelberg, Germany

${ }^{12}$ Indiana University, Department of Physics, Swain Hall West 117, Bloomington IN 47405, USA

${ }^{13}$ Queen Mary and Westfield College, University of London, London E1 4NS, UK

${ }^{15}$ University College London, London WC1E 6BT, UK

${ }^{16}$ Department of Physics, Schuster Laboratory, The University, Manchester M13 9PL, UK

${ }^{17}$ Department of Physics, University of Maryland, College Park, MD 20742, USA

${ }^{18}$ Laboratoire de Physique Nucléaire, Université de Montréal, Montréal, Quebec H3C 3J7, Canada

${ }^{19}$ University of Oregon, Department of Physics, Eugene OR 97403, USA

${ }^{20}$ Rutherford Appleton Laboratory, Chilton, Didcot, Oxfordshire OX11 0QX, UK

${ }^{21}$ CEA, DAPNIA/SPP, CE-Saclay, F-91191 Gif-sur-Yvette, France

${ }^{22}$ Department of Physics, Technion-Israel Institute of Technology, Haifa 32000, Israel

${ }^{23}$ Department of Physics and Astronomy, Tel Aviv University, Tel Aviv 69978, Israel

${ }^{24}$ International Centre for Elementary Particle Physics and Department of Physics, University of Tokyo, Tokyo 113, and Kobe University, Kobe 657, Japan

${ }^{25}$ Brunel University, Uxbridge, Middlesex UB8 3PH, UK

${ }^{26}$ Particle Physics Department, Weizmann Institute of Science, Rehovot 76100, Israel

${ }^{27}$ Universität Hamburg/DESY, II Institut für Experimental Physik, Notkestrasse 85, D-22607 Hamburg, Germany

${ }^{28}$ University of Victoria, Department of Physics, P O Box 3055, Victoria BC V8W 3P6, Canada

${ }^{29}$ University of British Columbia, Department of Physics, Vancouver BC V6T 1Z1, Canada

${ }^{30}$ University of Alberta, Department of Physics, Edmonton AB T6G 2J1, Canada

${ }^{31}$ Duke University, Dept of Physics, Durham, NC 27708-0305, USA

${ }^{32}$ Technische Hochschule Aachen, III Physikalisches Institut, Sommerfeldstrasse 26-28, D-52056 
Aachen, Germany

${ }^{a}$ Also at TRIUMF, Vancouver, Canada V6T 2 A3

${ }^{b}$ Royal Society University Research Fellow 


\section{Introduction}

The leptonic branching ratios of the tau lepton have been measured using data collected in 1991 and 1992 with the OPAL detector at LEP. These measurements, in conjunction with lepton masses and lifetimes, are used to determine the relative electroweak couplings of the electron, muon and tau. They are also used to make a direct test of electron-muon universality in tau decays and are extended to all three lepton flavours by comparing tau and muon decays. Finally, these measurements are used to calculate $\alpha_{s}\left(Q^{2}=M_{\tau}^{2}\right)$ which after $Q^{2}$ evolution is compared to the value of $\alpha_{s}\left(Q^{2}=M_{Z}^{2}\right)$ extracted from the $Z^{0}$ line shape analysis.

The tau event selections and decay mode identification cuts used in this analysis are similar to those used in an earlier OPAL measurement of $\tau$ leptonic branching ratios using the 1990 data [1]. We describe here the procedures used to make the branching ratio measurements and outline how backgrounds and efficiencies and their corresponding systematic errors are determined from Monte Carlo events and data control samples.

\section{The OPAL Detector}

OPAL is a general purpose detector covering the full solid angle with approximate cylindrical symmetry about the $\mathrm{e}^{+} \mathrm{e}^{-}$beam axis [2]. The coordinate system is defined with $+z$ along the $\mathrm{e}^{-}$beam direction. Polar and azimuthal angles are denoted by $\theta$ and $\phi$ respectively. This analysis is confined to the barrel region of the detector $|\cos \theta|<0.68$. The central tracking chambers, operating at a pressure of 4 bar, measure the momenta of charged particles over almost the entire solid angle in a uniform axial magnetic field of $0.435 \mathrm{~T}$. Located between the pressure vessel and a beryllium beam pipe is a two layer silicon strip vertex detector [3]. The innermost drift tracking chamber is a precision vertex chamber which is surrounded by a large volume jet drift chamber divided into 24 azimuthal sectors, each with a radial plane of 159 axial anode sense wires. The charge deposited on a wire provides a measurement of the energy loss $\mathrm{dE} / \mathrm{dx}$ of charged particles passing through the chamber. In the region $|\cos \theta|<0.72$, the jet chamber is surrounded by a cylindrical array of 192 planar drift chambers which provide a precise measurement of the $z$ coordinates of charged particles as they leave the jet chamber.

In the barrel region the central tracking chambers are surrounded by a time-of-flight (TOF) counter array consisting of 160 scintillator bars with photomultiplier readout at both ends, followed by an electromagnetic calorimeter (ECAL) with a presampler. In addition to the barrel calorimeter, covering $|\cos \theta|<0.82$, two endcap calorimeters extend coverage to $|\cos \theta|<0.98$. The barrel part consists of 9440 lead-glass blocks, each 24.6 radiation lengths deep and having a cross section of $10 \times 10 \mathrm{~cm}^{2}$ at a radius of $2.4 \mathrm{~m}$. The barrel presampler, which measures electromagnetic showers originating in the magnetic coil, consists of two layers of limitedstreamer tubes with $1 \mathrm{~cm}$ pitch wire and cathode-strip readout. The instrumented magnet return yoke serves as a hadron calorimeter (HCAL) and muon tracker, consisting in the barrel of up to 9 layers of limited streamer tubes sandwiching $10 \mathrm{~cm}$ layers of iron, with inductive readout of the tubes onto large pads and onto $4 \mathrm{~mm}$ wide aluminium strips. The detector is surrounded by four layers of (MUON) drift chambers for the detection of muons emerging from the hadron calorimeter. 
Data used in this analysis were recorded in 1991 and 1992. The 1991 data, corresponding to an integrated luminosity of $14.0 \mathrm{pb}^{-1}$, were taken at a number of centre of mass energies $\left(\mathrm{E}_{\mathrm{cm}}\right)$ between 88.5 and $93.8 \mathrm{GeV}$ with about $65 \%$ taken at the peak of the $\mathrm{Z}^{0}$ resonance. The $25.1 \mathrm{pb}^{-1}$ of data collected in 1992 were recorded at the peak energy, $\mathrm{E}_{\mathrm{cm}}=91.299 \mathrm{GeV}$.

For Monte Carlo studies the OPAL detector response is simulated by a program [4] that treats in detail the detector geometry and material, as well as effects of detector resolution and efficiencies. Nonetheless, it is necessary to apply additional, random Gaussian smearing to Monte Carlo reconstructed shower energies and inverse track momenta in order to reproduce the resolutions measured in the data.

\section{Selection of events}

At LEP $\tau$ pair events are easier to distinguish from backgrounds than at lower energy colliders. In addition the high degree of redundancy in the hardware and software triggers [5], [6] allows the trigger efficiency to be measured from the data. The trigger efficiency for $\tau$ pair events is close to $100 \%$.

For an event to be considered in the $\mathrm{e}^{+} \mathrm{e}^{-} \rightarrow \tau^{+} \tau^{-}$preselection the detectors in the barrel region and their associated triggers are required to be fully operational. A charged track must have a minimum momentum transverse to the beam of $100 \mathrm{MeV}$, a measured $\left|d_{0}\right|<2 \mathrm{~cm}$, and a measured $\left|z_{0}\right|<75 \mathrm{~cm}$, where $\left|d_{0}\right|$ is the distance of closest approach of the track to the beam axis, and $\left|z_{0}\right|$ is the displacement along the beam axis from the nominal interaction point at the point of closest approach to the beam. The track must also have at least 20 measured space points in the jet chamber and at least one point within $75 \mathrm{~cm}$ of the beam axis.

\section{$3.1 \quad \mathrm{e}^{+} \mathrm{e}^{-} \rightarrow \tau^{+} \tau^{-}$}

The selection of $\mathrm{e}^{+} \mathrm{e}^{-} \rightarrow \tau^{+} \tau^{-}$events is made by first rejecting beam gas, cosmic ray and other background events and then rejecting multihadronic and two photon events. After this preselection, only lepton pair events should remain and then finally the $\mathrm{e}^{+} \mathrm{e}^{-} \rightarrow \mathrm{e}^{+} \mathrm{e}^{-}$and $\mathrm{e}^{+} \mathrm{e}^{-} \rightarrow \mu^{+} \mu^{-}$events are rejected. In selecting $\tau$ pair events and in identifying $\tau$ decay channels, both charged tracks and electromagnetic clusters, where a cluster is defined as a group of contiguous lead-glass blocks which has a minimum energy of $100 \mathrm{MeV}$, are considered.

The multihadron background is reduced by requiring between 2 and 6 charged tracks and excluding events with more than 10 ECAL clusters. Cosmic ray backgrounds are removed by requiring at least one charged track with a measured $\left|d_{0}\right|<0.5 \mathrm{~cm}$ and a measured $\left|z_{0}\right|<20$ $\mathrm{cm}$, and requiring the magnitude of the average $z$ of all tracks at their points of closest approach to the beam to be less than $20 \mathrm{~cm}$. In addition, there must be at least one TOF signal within $10 \mathrm{~ns}$ of the nominal expected value. An event is rejected if all pairs of TOF signals separated by more than $165^{\circ}$ in azimuth have time differences greater than $10 \mathrm{~ns}$.

Using a jet finding algorithm, charged tracks and clusters are associated to cones of halfangle $35^{\circ}[1]$. A candidate lepton pair event must have exactly two cones, and each cone must 
contain at least one charged track. The tracks and clusters inside the cone form the jets. The visible energy of each jet is taken as the maximum of the sum of the track momenta and the ECAL cluster energy associated to that jet. Only cones with visible energy greater than $1 \%$ of

the beam energy are accepted. The average value of $|\cos \theta|$ for the two charged cones must be less than 0.68 .

To remove backgrounds due to two-photon processes, the acollinearity of the two jets is required to be less than $15^{\circ}$, where the directions of the jets are given by the momentum sums of the tracks and clusters. Rejection of residual two-photon backgrounds exploits the low visible energies and very low transverse momenta typical of $\mathrm{e}^{+} \mathrm{e}^{-} \rightarrow\left(\mathrm{e}^{+} \mathrm{e}^{-}\right) X$ events. An event is rejected if the sum of visible energies of the jets is less than $3 \%$ of $\mathrm{E}_{\mathrm{cm}}$. Further, if the total visible energy is less than $20 \%$ of $\mathrm{E}_{\mathrm{cm}}$, the event is rejected if the missing transverse momenta, calculated separately for charged tracks and for ECAL clusters, are both less than $2 \mathrm{GeV}$.

The $\mathrm{e}^{+} \mathrm{e}^{-} \rightarrow \mathrm{e}^{+} \mathrm{e}^{-}$background in the $\tau^{+} \tau^{-}$sample is reduced substantially by requiring that events satisfy either $\sum \mathrm{E}_{\text {ecal }} \leq 0.7 \mathrm{E}_{\mathrm{cm}}$ or $\left(\sum \mathrm{E}_{\text {ecal }}+0.3 \sum \mathrm{p}_{\mathrm{trk}}\right) \leq \mathrm{E}_{\mathrm{cm}}$, where $\sum \mathrm{E}_{\text {ecal }}$ is the total energy deposited in the lead-glass calorimeter and $\sum p_{\text {trk }}$ is the total momentum of the charged tracks. Muon pair events are removed by rejecting events where both cones contain a particle identified as a muon by one of the detectors (ECAL, HCAL and MUON), as detailed in the discussion of the $\tau \rightarrow \mu \nu \bar{\nu}$ selection, and the scalar sum of the highest energy cluster and the highest momentum track when summed for both cones is greater than $0.6 \mathrm{E}_{\mathrm{cm}}$. These cuts to remove $\mathrm{e}^{+} \mathrm{e}^{-} \rightarrow \mathrm{e}^{+} \mathrm{e}^{-}$and $\mathrm{e}^{+} \mathrm{e}^{-} \rightarrow \mu^{+} \mu^{-}$events are similar to those used in [1], except that the $\mathrm{e}^{+} \mathrm{e}^{-} \rightarrow \mathrm{e}^{+} \mathrm{e}^{-}$rejection cuts have been tightened, thus decreasing the $\mathrm{e}^{+} \mathrm{e}^{-} \rightarrow \mathrm{e}^{+} \mathrm{e}^{-}$background in both $\mathrm{e}^{+} \mathrm{e}^{-} \rightarrow \tau^{+} \tau^{-}$and $\tau \rightarrow \mathrm{e} \nu \bar{\nu}$ samples. The distributions of track variables on which cuts are made are consistent between the 1991 and 1992 data. Applying these criteria to the complete 1991 and 1992 data samples gives $27196 \mathrm{e}^{+} \mathrm{e}^{-} \rightarrow \tau^{+} \tau^{-}$candidate events, consisting of 8151 events from the 1991 sample and 19045 events from the 1992 sample.

In the following sections the selection cuts for $\tau \rightarrow \mathrm{e} \nu \bar{\nu}$ and $\tau \rightarrow \mu \nu \bar{\nu}$ are described. The data and Monte Carlo distributions agree reasonably well in all the variables that are used in these selections. However as control samples from the data are used in this analysis to estimate efficiencies and backgrounds, the results are not very sensitive to this agreement and so only a few representative distributions are presented.

\section{$3.2 \tau \rightarrow \mathrm{e} \nu \bar{\nu}$}

Further cuts are imposed on the $\mathrm{e}^{+} \mathrm{e}^{-} \rightarrow \tau^{+} \tau^{-}$candidate events in order to isolate a clean sample of $\tau \rightarrow \mathrm{e} \nu \bar{\nu}$ decays. The number of charged tracks assigned to the candidate cone must be less than or equal to two. Most electron events will have one charged track but the cut is set at two to retain some of those events where one of the tracks of a converting photon is also reconstructed. If there are two tracks assigned to a cone, the higher momentum track is taken as the electron candidate. The distribution of the ratio of the energy of the cluster associated to the electron candidate $\left(\mathrm{E}_{\mathrm{cls}}\right)$ and the candidate track's reconstructed momentum, $\mathrm{E}_{\mathrm{cls}} / \mathrm{p}_{\text {trk }}$, is shown in Figure 1(a). The electron signal is enhanced by the selection $0.6<\mathrm{E}_{\text {cls }} / \mathrm{p}_{\text {trk }}<2.0$. The ECAL cluster energies used in the $\tau \rightarrow \mathrm{e} \nu \bar{\nu}$ selection are corrected for the expected energy loss of electrons in the coil preceding the calorimeter. The distribution of the number 
of lead-glass blocks containing at least $90 \%$ of the cluster energy, $\mathrm{N}_{\text {blk }}^{90}$, is shown in Figure 1(b). Electron showers are enhanced by demanding $\mathrm{N}_{\mathrm{blk}}^{90} \leq 3$.

In order to reduce background from hadronic $\tau$ decays, several cuts are made on the distribution of energy in the calorimeters. First the excess energy, $\mathrm{E}_{\text {excess }}$, is required to be less than $0.02 \mathrm{E}_{\mathrm{cm}}$ where $\mathrm{E}_{\text {excess }}$ is the total electromagnetic cluster energy assigned to the cone, excluding any cluster associated to the electron candidate track. $\tau \rightarrow$ e $\nu \bar{\nu}$ decays have generally small values of $\mathrm{E}_{\text {excess }}$ while background (mainly from hadronic $\tau$ decays with neutral pions) has a flatter distribution of excess energy. Rejection of hadronic tau decays with neutral pions is also assisted by using the good spatial resolution of the barrel presampler. A cut is made on the difference in azimuth, $\delta \phi_{\max }$, between the track and the presampler cluster farthest away in azimuth but still assigned to the cone. It is required that $\delta \phi_{\max }<5^{\circ}$. Tracks are also rejected if they point to inactive regions of the presampler or electromagnetic calorimeter. Finally, backgrounds from $\tau \rightarrow \mu \nu \bar{\nu}$ and $\tau \rightarrow \pi(\mathrm{K}) \nu$ are reduced, as can be seen in Figure 2(a), by excluding cones with associated activity in the hadronic calorimeter (HCAL). Specifically, the

number of HCAL layers, $\mathrm{N}_{\text {layers }}^{\mathrm{HC}}$, containing a signal assigned to the $\tau$ cone is required to be no more than one.

In order to remove a small residual contamination from $\mathrm{e}^{+} \mathrm{e}^{-} \rightarrow \mathrm{e}^{+} \mathrm{e}^{-}$events where the energy of one electron is mismeasured, an electron candidate is rejected if the opposite cone consists of a single charged particle of momentum, $\mathrm{p}_{\text {trk }}$, greater than $0.75 \mathrm{E}_{\text {beam }}$ whenever the acoplanarity angle, $\theta_{\text {Acop }}$ between the electron candidate track and the opposite track is less than $0.1^{\circ}$. The acoplanarity is defined as the acollinearity between the tracks in each cone in the plane transverse to the beam.

Finally, in order to ensure reliable electron identification with low background and well understood efficiency, the electron shower energy must satisfy $\mathrm{x}_{\mathrm{e}} \equiv \mathrm{E}_{\text {cone }} / \mathrm{E}_{\text {beam }}>0.05$ where $\mathrm{E}_{\text {cone }}$ is the total ECAL energy in the cone (see Figure 2(b)). We note the small surplus of events at $\mathrm{x}_{\mathrm{e}} \approx 1$, suggesting a slight, remnant background from $\mathrm{e}^{+} \mathrm{e}^{-} \rightarrow \mathrm{e}^{+} \mathrm{e}^{-}$events in the $\tau \rightarrow \mathrm{e} \nu \bar{\nu}$ sample. This background is discussed in section 4 .

After the full selection is carried out, $7322 \tau \rightarrow \mathrm{e} \nu \bar{\nu}$ candidates are selected from the combined 1991 and 1992 data samples.

\section{$3.3 \tau \rightarrow \mu \nu \bar{\nu}$}

The following cuts are imposed on the $\mathrm{e}^{+} \mathrm{e}^{-} \rightarrow \tau^{+} \tau^{-}$candidate decays in order to isolate a sample of $\tau \rightarrow \mu \nu \bar{\nu}$ events with low background. The number of charged tracks assigned to the candidate cone must be equal to one. Most $\tau \rightarrow \mu \nu \bar{\nu}$ decays within the detector acceptance are characterised by a small energy deposition in the ECAL and associated signals in the HCAL and MUON subdetectors consistent with the passage of a penetrating, minimum-ionising particle. In order to accept muons that either enter inactive regions of the HCAL or MUON subdetectors or are accompanied by photon radiation, a $\tau \rightarrow \mu \nu \bar{\nu}$ candidate must satisfy at least two of the following three requirements:

1. Identification by the electromagnetic calorimeter[ECID]: $\mathrm{E}_{\mathrm{cls}}<2 \mathrm{GeV}$ where $\mathrm{E}_{\text {cls }}$ is the cluster energy associated to the candidate track. 
2. Identification by the hadron calorimeter [HCID]: $\mathrm{N}_{\text {layers }}^{\mathrm{HC}} \geq 4$ where $\mathrm{N}_{\text {layers }}^{\mathrm{HC}}$ is the number of HCAL layers containing signals associated to the track (see Figure 3(a)).

3. Identification by the MUON chamber layers [MUID]: $\mathrm{N}_{\text {layers }}^{\mathrm{MU}} \geq 2$ where $\mathrm{N}_{\text {layers }}^{\mathrm{MU}}$ is the total number of layers with signals in the MUON detector associated to the track (see Figure $3(\mathrm{~b}))$.

Any signals in the HCAL associated with a charged track must be consistent with the passage of a minimum-ionising particle, even when the HCID condition is not satisfied. We define $\mathrm{N}_{\text {hits/layer }}^{\mathrm{HC}}$ as the mean number of hit strips which have been associated with a charged track, averaged over all the layers of the HCAL which show activity. A $\tau \rightarrow \mu \nu \bar{\nu}$ candidate is rejected if $\mathrm{N}_{\text {hits/layer }}^{\mathrm{HC}} \geq 3$. Muon candidates produce 1 or 2 hits per layer on average.

Residual background from hadronic $\tau$ decays accompanied by $\pi^{0}$ production is suppressed by requiring $\mathrm{M}_{\text {trk-ecal }}<0.3 \mathrm{GeV}$ where $\mathrm{M}_{\text {trk-ecal }}$ is the invariant mass of the charged track (assuming that the track has a mass of a $\pi^{ \pm}$) and all ECAL clusters in the cone (assuming a $\gamma$ hypothesis). In the calculation, $0.5 \mathrm{GeV}$ is subtracted from the energy of the ECAL cluster nearest the charged track, to account for the average energy deposition from a minimum-ionising particle. This invariant mass quantifies not only the angular spread of multiple clusters within a cone but also the angular difference between the track direction and the centroid of its associated ECAL cluster.

In order to suppress residual $\mathrm{e}^{+} \mathrm{e}^{-} \rightarrow \mu^{+} \mu^{-}$contamination, a $\tau \rightarrow \mu \nu \bar{\nu}$ candidate is rejected if the opposite cone consists of exactly one charged track, consistent with being a muon and with $\mathrm{x}_{\mu} \equiv\left(\mathrm{p}_{\text {trk }}+\mathrm{E}_{\mathrm{cls}}-0.5 \mathrm{GeV}\right) / \mathrm{E}_{\mathrm{beam}} \geq 0.8$ where the ECAL cluster energy, $\mathrm{E}_{\mathrm{cls}}$, is added to the track momentum in order to reduce sensitivity to radiated photons. A track is considered to be consistent with a muon if it satisfies any one of the following three criteria: identification according to either MUID or HCID as defined above or the track passes through a geometric region where neither the hadron calorimeter nor the MUON chambers are fully active.

A further rejection on $\mathrm{e}^{+} \mathrm{e}^{-} \rightarrow \mu^{+} \mu^{-}$background is made by rejecting candidate events if both cones contain a high momentum particle. A candidate is rejected if the opposite cone contains a track with $0.9<\mathrm{x}_{\mu}<1.1$ and the near side track has a $\mathrm{x}_{\mu}>0.9$. The azimuthal angle, $\phi_{\text {trk }}$, is plotted in Figure 4(a) for all candidate $\tau \rightarrow \mu \nu \bar{\nu}$ tracks with $\mathrm{x}_{\mu}>0.6$. The region near the anodes $\left(7.5^{\circ}\right)$ is not modelled accurately by the Monte Carlo and would introduce a large uncertainty in the background fraction of $\tau \rightarrow \mu \nu \bar{\nu}$ events. The surplus events in the data are taken to be $\mathrm{e}^{+} \mathrm{e}^{-} \rightarrow \mu^{+} \mu^{-}$background in the $\tau$ pair sample. In these events one or both muons travel near an anode wire plane in the jet chamber where there is some degradation of reconstructed momentum resolution, causing a small fraction of the $\mathrm{e}^{+} \mathrm{e}^{-} \rightarrow \mu^{+} \mu^{-}$events to fall below the $0.6 \mathrm{E}_{\mathrm{cm}}$ cut and hence not be rejected as dimuon events (see section 3.1). To avoid this uncertainty, $\tau \rightarrow \mu \nu \bar{\nu}$ candidates with $\mathrm{x}_{\mu}>0.6$ must satisfy $\left|\phi_{\text {trk }}-\phi_{\text {anode }}\right|>0.5^{\circ}$ where $\phi_{\text {trk }}$ is the azimuthal direction of the charged track at its closest approach to the beam and $\phi_{\text {anode }}$ is the azimuthal angle of the anode plane of the jet chamber sector, traversed by the track.

Finally, in order to ensure reliable muon identification with low background and well understood efficiency, the muon candidates must satisfy $x_{\mu}>0.05$. Figure 4(b) shows that this 
cut entails the removal of only a small fraction of data. After the complete selection, 7941 $\tau \rightarrow \mu \nu \bar{\nu}$ candidates are selected from the 1991 and 1992 data samples.

\section{Systematic Uncertainties}

\subsection{Backgrounds}

The residual background fractions in the $\mathrm{e}^{+} \mathrm{e}^{-} \rightarrow \tau^{+} \tau^{-}, \tau \rightarrow \mathrm{e} \nu \bar{\nu}$ and $\tau \rightarrow \mu \nu \bar{\nu}$ samples are estimated from Monte Carlo studies ( with full detector simulation ) of $\mathrm{e}^{+} \mathrm{e}^{-} \rightarrow \mathrm{e}^{+} \mathrm{e}^{-}[7]$, $\mathrm{e}^{+} \mathrm{e}^{-} \rightarrow \mu^{+} \mu^{-}[8], \mathrm{e}^{+} \mathrm{e}^{-} \rightarrow q \bar{q}[9], \mathrm{e}^{+} \mathrm{e}^{-} \rightarrow\left(\mathrm{e}^{+} \mathrm{e}^{-}\right) X[10]$ and all decay modes of the reaction $\mathrm{e}^{+} \mathrm{e}^{-} \rightarrow \tau^{+} \tau^{-}$[11]. The backgrounds estimated from Monte Carlo are compared with those obtained from background enhanced samples of data. The ratio of the numbers of events from data and Monte Carlo from a particular background source is calculated. When there is good agreement between data and Monte Carlo the Monte Carlo estimate is used and the statistical error of the comparison is used as a systematic error. When there is disagreement from unity at the $95 \%$ confidence level, the Monte Carlo estimate is scaled to restore equality with the data and, to reflect the initial disagreement, the systematic error is increased by half the discrepancy from unity. In most cases the data and Monte Carlo agree, giving confidence that the Monte Carlo models the background correctly when extrapolated into the signal region. Details of the cases where the data and Monte Carlo disagree are now described.

The contamination in the tau pair sample due to $\mathrm{e}^{+} \mathrm{e}^{-} \rightarrow \mathrm{e}^{+} \mathrm{e}^{-}$is checked by studying the number of events identified as electron pairs with total cluster energy, $\sum \mathrm{E}_{\text {ecal }}$, between 0.7 and $0.85 \mathrm{E}_{\mathrm{cm}}$ (see Figure 5(a)). The number of events passing these cuts is calculated for the data and for all Monte Carlo sources (normalised to the data luminosity). The number of events from sources other than $\mathrm{e}^{+} \mathrm{e}^{-} \rightarrow \mathrm{e}^{+} \mathrm{e}^{-}$is determined by Monte Carlo and is subtracted from the measured number of events in this region to give the estimated number of $\mathrm{e}^{+} \mathrm{e}^{-} \rightarrow \mathrm{e}^{+} \mathrm{e}^{-}$events in the data. This is then compared with the number of events predicted by the $\mathrm{e}^{+} \mathrm{e}^{-} \rightarrow \mathrm{e}^{+} \mathrm{e}^{-}$ Monte Carlo, giving a ratio $\operatorname{DATA}\left(\mathrm{e}^{+} \mathrm{e}^{-} \rightarrow \mathrm{e}^{+} \mathrm{e}^{-}\right) / \mathrm{MC}\left(\mathrm{e}^{+} \mathrm{e}^{-} \rightarrow \mathrm{e}^{+} \mathrm{e}^{-}\right)=1.81 \pm 0.26$. Since a discrepancy is seen within this control sample, due to imperfect modelling of the tails of the electromagnetic calorimeter response to electrons, the predicted background fraction from $\mathrm{e}^{+} \mathrm{e}^{-} \rightarrow \mathrm{e}^{+} \mathrm{e}^{-}$in the $\tau$ pair sample is modified from the Monte Carlo estimate of $(0.13 \pm 0.03) \%$. The uncertainty on the correction factor is increased from 0.26 (the statistical error) to 0.41 , half the difference between the measured background correction factor and unity. It should be noted that since the predicted Monte Carlo background is small, this is not a dominant error. The corrected background estimate for the process $\mathrm{e}^{+} \mathrm{e}^{-} \rightarrow \mathrm{e}^{+} \mathrm{e}^{-}$is then $(0.13 \pm 0.03) *(1.81 \pm 0.41)=$ $(0.24 \pm 0.07) \%$.

The accuracy of the prediction of the muon pair background in the tau pair candidates is assessed by comparing the number of events with both cones identified as muons by at least two of the three muon identification schemes ECID, HCID and MUID described earlier. In addition the scalar sum of the highest energy cluster and the highest momentum track of both cones is required to be between 0.6 and $0.75 \mathrm{E}_{\mathrm{cm}}$ (Figure $5(\mathrm{~b})$ ). Using this control sample we find the ratio $\operatorname{DATA}\left(\mathrm{e}^{+} \mathrm{e}^{-} \rightarrow \mu^{+} \mu^{-}\right) / \mathrm{MC}\left(\mathrm{e}^{+} \mathrm{e}^{-} \rightarrow \mu^{+} \mu^{-}\right)=2.22 \pm 0.30$, indicating that there is an underestimate of the background from $\mathrm{e}^{+} \mathrm{e}^{-} \rightarrow \mu^{+} \mu^{-}$in the tau pair sample which is due 
to the imperfect Monte Carlo modelling of tracks near the jet chamber anode wires. Both the predicted background fraction and its associated systematic error are corrected by a factor of $2.22 \pm 0.61$. This yields a corrected background of $(0.45 \pm 0.02) *(2.22 \pm 0.61)=(1.00 \pm 0.28) \%$. This problem is avoided in the $\tau \rightarrow \mu \nu \bar{\nu}$ sample by excluding track candidates of high $\mathrm{x}_{\mu}$ which travel close to an anode plane.

The fraction of $\tau \rightarrow \pi(\mathrm{K}) \nu$ background in the $\tau \rightarrow$ e $\nu \bar{\nu}$ sample is determined by Monte Carlo to be $(3.99 \pm 0.09) \%$. In order to check the Monte Carlo simulation of the background, an enhanced sample of $\tau \rightarrow \pi(\mathrm{K}) \nu$ decays is selected by demanding that $\tau \rightarrow$ e $\nu \bar{\nu}$ candidates have $0.5<\mathrm{E}_{\text {cls }} / \mathrm{p}_{\text {trk }}<0.9$ and $\left(\mathrm{dE} / \mathrm{dx}_{\text {meas }}-\mathrm{dE} / \mathrm{dx}_{\mathrm{e}}\right)<-1.5 \sigma$ where $\mathrm{dE} / \mathrm{dx}_{\text {meas }}$ is the measured energy loss, and $\mathrm{dE} / \mathrm{dx}_{\mathrm{e}}$ and $\sigma$ are the expected energy loss for an electron and its error. The Monte Carlo and data $\mathrm{E}_{\text {cls }} / \mathrm{p}_{\text {trk }}$ distributions for these events are shown in Figure 6(a). This sample contains over $60 \%$ of the $\tau \rightarrow \pi(\mathrm{K}) \nu$ background seen in the selected $\tau \rightarrow$ e $\nu \bar{\nu}$ data. For cones satisfying the above cuts, we find : $\operatorname{DATA}(\tau \rightarrow \pi(\mathrm{K}) \nu) / \mathrm{MC}(\tau \rightarrow \pi(\mathrm{K}) \nu)=0.63 \pm 0.06$. The discrepancy, due to imperfect modelling of the calorimeter response to hadrons, is corrected by reducing the background estimate and adjusting its error accordingly: $(3.99 \pm 0.09) *(0.63 \pm$ $0.19)=(2.51 \pm 0.74) \%$.

In order to check the contamination from $\tau \rightarrow \pi(\mathrm{K}) \nu$ in the $\tau \rightarrow \mu \nu \bar{\nu}$ sample, the number of cones identified simultaneously as both $\tau \rightarrow \mu \nu \bar{\nu}$ and $\tau \rightarrow \pi(\mathrm{K}) \nu[1]$ is calculated for data and Monte Carlo, giving a ratio $\operatorname{DATA}(\tau \rightarrow \pi(\mathrm{K}) \nu) / \mathrm{MC}(\tau \rightarrow \pi(\mathrm{K}) \nu)=0.59 \pm 0.11$. The Monte Carlo and data $\mathrm{N}_{\text {hits/layer }}^{\mathrm{HC}}$ distributions for these events are shown in Figure 6(b). The discrepancy between data and Monte Carlo is due to the imperfect modelling of hadronic showers in the simulation. The background contamination from $\tau \rightarrow \pi(\mathrm{K}) \nu$ in the $\tau \rightarrow \mu \nu \bar{\nu}$ sample is corrected accordingly and its systematic error is increased to take this into account yielding $(2.23 \pm 0.07) *(0.59 \pm 0.21)=(1.32 \pm 0.46) \%$.

The final corrected backgrounds in the $\tau$ pair,$\tau \rightarrow$ e $\nu \bar{\nu}$ and $\tau \rightarrow \mu \nu \bar{\nu}$ samples are shown in Table 1 together with the original Monte Carlo predictions.

\subsection{Efficiency correction factors}

The selection efficiency in each momentum bin is taken initially from Monte Carlo estimates which are shown in Figure 7. It is corrected with factors derived from comparisons of the data and Monte Carlo control samples of electrons and muons at low x (using two-photon events) and at $\mathrm{x} \approx 1$ (using Bhabha or dimuon events). The efficiency correction factor is defined as the ratio of the fraction of real data to the fraction of Monte Carlo data passing the same sets of cuts.

The control samples are initially selected by making loose requirements on global event variables. The samples are purified by making strict particle identification requirements on the cone opposite the cone under study. If the event passes the opposite cone cuts, then the near side cone is considered to contain a candidate electron or muon and is used for the efficiency measurement. 


\subsection{1 $\tau \rightarrow \mathrm{e} \nu \bar{\nu}$}

The $\mathrm{e}^{+} \mathrm{e}^{-} \rightarrow \mathrm{e}^{+} \mathrm{e}^{-}$control sample is composed of events identified as electron pairs using the total cluster and track energies as described in section 3.1. One cone in the event (the "opposite cone") must contain a track with $\mathrm{x}_{\mathrm{e}}>0.8$ and must satisfy the $\tau \rightarrow$ e $\nu \bar{\nu}$ selection requirements on the variables $\mathrm{E}_{\text {cls }} / p_{\text {trk }}, \mathrm{E}_{\text {excess }}, \delta \phi_{\max }, \mathrm{N}_{\text {layers }}^{\mathrm{HC}}, \mathrm{N}_{\mathrm{blk}}^{90}$, and the ECAL geometrical acceptance cut. No requirements are made on the other cone in the event (the "near cone"). The $\mathrm{e}^{+} \mathrm{e}^{-} \rightarrow\left(\mathrm{e}^{+} \mathrm{e}^{-}\right) \mathrm{e}^{+} \mathrm{e}^{-}$control sample is obtained by selecting non-lepton pair events with two cones and one charged track per cone. The same $\tau \rightarrow$ e $\nu \bar{\nu}$ selection requirements as for the $\mathrm{e}^{+} \mathrm{e}^{-} \rightarrow \mathrm{e}^{+} \mathrm{e}^{-}$control sample are made on the opposite cone except that the track is required to have $\mathrm{dE} / \mathrm{dx}>9.5 \mathrm{keV} / \mathrm{cm}$, consistent with that of an electron, and $\mathrm{x}_{\mathrm{e}}<0.25$. These selection cuts result in high purity unbiased control samples.

Outside the $\mathrm{x}_{\mathrm{e}}$ regions of the control samples, a linear interpolation is made between the correction factor for the two-photon electrons with $0.05 \leq \mathrm{x}_{e} \leq 0.15$ (measured to be $0.969 \pm 0.009$ ) and the correction factor for the Bhabha electrons with $0.85 \leq \mathrm{x}_{\mathrm{e}} \leq 1.00$ (measured to be $1.000 \pm 0.003)$.

For the branching ratio calculation, a single global efficiency correction factor is calculated using the bin-by-bin factors and the Monte Carlo $\mathrm{x}_{\mathrm{e}}$ distribution. This global correction factor is $0.981 \pm 0.007 \pm 0.009$, where the first error comes from the statistical error of the two photon and Bhabha comparisons and the second error is the systematic uncertainty on the interpolation of the corrections between low and high momenta. In practice, we take the difference between the correction factors, determined at low and high $\mathrm{x}$, divided by $\sqrt{12}$ as the estimate of the systematic error. This assumes that the two efficiency correction measurements are sampled from a uniform distribution and makes no additional assumptions about their energy dependence.

The uncorrected efficiency for selecting $\tau \rightarrow \mathrm{e} \nu \bar{\nu}$ within the $|\cos \theta| \leq 0.68$ acceptance is estimated from the Monte Carlo sample to be $\epsilon_{\text {uncorr }}^{e}=(72.7 \pm 0.2) \%$. After applying the correction factor described above, the final selection efficiency is $\epsilon_{\text {corr }}^{e}=(71.3 \pm 0.9) \%$ (see Table 2).

\subsection{2 $\tau \rightarrow \mu \nu \bar{\nu}$}

The $\mathrm{e}^{+} \mathrm{e}^{-} \rightarrow \mu^{+} \mu^{-}$control sample is obtained by requiring that the event is identified as a muon pair using the total cluster and track energies and the loose muon identification requirement described in section 3.1. The opposite cone must satisfy the $\tau \rightarrow \mu \nu \bar{\nu}$ selection requirements on the variables $\mathrm{M}_{\text {trk-ecal }}$ and $\mathrm{N}_{\text {hits/layer }}^{\mathrm{HC}}$ described in section 3.3 and must be identified as a muon having $\mathrm{x}_{\mu}>0.8$ by at least two of the three muon detection schemes (ECID, HCID, MUID). No further requirements are imposed on the near cone. The $\mathrm{e}^{+} \mathrm{e}^{-} \rightarrow\left(\mathrm{e}^{+} \mathrm{e}^{-}\right) \mu^{+} \mu^{-}$ control sample is obtained by selecting non lepton pair events with two cones. Each cone must contain one track with $\mathrm{dE} / \mathrm{dx}<8.5 \mathrm{keV} / \mathrm{cm}$, and the opposite cone is required to satisfy the same $\tau \rightarrow \mu \nu \bar{\nu}$ selection requirements as the $\mathrm{e}^{+} \mathrm{e}^{-} \rightarrow \mu^{+} \mu^{-}$control sample but with a low momentum track, $\mathrm{x}_{\mu}<0.25$.

The efficiency correction factor is $0.973 \pm 0.012$ for the two-photon sample at low $\mathrm{x}_{\mu}$ and 
$0.995 \pm 0.002$ for dimuons at high $\mathrm{x}_{\mu}$. By interpolation, the efficiency correction factor for the $\tau \rightarrow \mu \nu \bar{\nu}$ data is $0.980 \pm 0.009 \pm 0.006$. As for the $\tau \rightarrow$ e $\nu \bar{\nu}$ data, the systematic uncertainties are comparable to the statistical errors, a reflection of the difference in the correction factors obtained at low and high $\mathrm{x}_{\mu}$.

The total uncorrected efficiency for selecting $\tau \rightarrow \mu \nu \bar{\nu}$ within the $|\cos \theta| \leq 0.68$ acceptance is estimated from the Monte Carlo sample to be $\epsilon_{\text {uncorr }}^{\mu}=(85.7 \pm 0.2) \%$. After applying the correction factor, the final selection efficiency is $\epsilon_{\text {corr }}^{\mu}=(84.0 \pm 0.9) \%$ (see Table 2$)$.

\section{$5 \quad$ Branching Ratio Results}

The branching ratios are calculated from the following expression:

$$
B(\tau \rightarrow \ell \nu \bar{\nu}) \equiv \frac{N_{\text {Cand }}^{\tau \rightarrow \ell \nu \bar{\nu}}}{N_{\text {Cand }}^{\tau}} \cdot \frac{\left(1-f_{B k g d}^{\tau \rightarrow \ell \bar{\nu}}\right)}{\left(1-f_{B k g d}^{\tau}\right)} \cdot \frac{1}{\epsilon^{\tau \rightarrow \ell \nu \bar{\nu}}} \cdot \frac{1}{F_{\text {Bias }}^{\tau \rightarrow \ell \nu \bar{\nu}}}
$$

The symbols have the following definitions: $\ell=e, \mu ; N_{C a n d}^{\tau \rightarrow \ell \bar{\nu}}$ and $N_{C a n d}^{\tau}$ are the number of $\tau \rightarrow \ell \nu \bar{\nu}$ and $\tau$ candidates respectively; $f_{B k g d}^{\tau}$ is the estimated contamination of non- $\tau$ events in the $\tau$ pair sample; $f_{B k g d}^{\tau \rightarrow l} \bar{\nu}$ is the estimated background fraction in the $\tau \rightarrow \ell \nu \bar{\nu}$ sample from other $\tau$ decay modes and non- $\tau$ sources; $\epsilon^{\tau \rightarrow \ell \nu \bar{\nu}}$ is the estimated efficiency for selecting $\tau \rightarrow \ell \nu \bar{\nu}$ decays from the preselected $\tau$ pair sample. The absolute efficiency for selecting $\tau$ pair candidates does not enter the expression but the effect of any efficiency difference between decay modes has to be included. $F_{B i a s}^{\tau \rightarrow \ell \nu}$ is the relative enhancement for detecting $\tau \rightarrow \ell \nu \bar{\nu}$ decays with respect to other $\tau$ decay modes. This was obtained by measuring the effect of the $\tau$ pair selection cuts on the relative numbers of each type of $\tau$ decay in Monte Carlo.

Results of the analysis are given in Table 3 yielding the branching ratio values $B(\tau \rightarrow \mathrm{e} \nu \bar{\nu})=(18.14 \pm 0.20 \pm 0.28) \%$ and $B(\tau \rightarrow \mu \nu \bar{\nu})=(17.48 \pm 0.18 \pm 0.23) \%$ where the first error is statistical and the second systematic. The estimated systematic errors are calculated from the errors on the backgrounds, efficiencies and bias factors listed in Table 3. The error on the bias factor is the statistical error from the Monte Carlo determination. A breakdown of the different contributions to the systematic error is shown in Table 4. In both channels, the systematic uncertainties and the statistical errors are of comparable magnitude and the dominant contribution to both the systematic errors is from the efficiency measurement. The main cause of this uncertainty is the energy dependence of the efficiency correction and the limited statistics in the two-photon control sample.

The branching ratios are calculated for the 1991 and 1992 data samples separately and are in good agreement. A number of other checks are also made in order to verify the systematic error estimates included in the earlier sections. The analysis has been repeated with modifications to the Monte Carlo smearing. The magnitude of the smearing has been varied to $50 \%$ and $150 \%$ of its optimised value and the central values of the leptonic branching ratios are changed by less than 0.0005 . A number of the cuts used to define the final electron and muon samples are modified and the efficiencies, background and branching ratios are recalculated. This check is performed for the variables with the largest discrepancy between Monte Carlo and data. Again the variation in branching ratios are small compared to the quoted errors. 
To check the stability of the results to the technique used to determine background, the correction method is altered. The actual values of the correction factors, whether or not they are consistent with unity at the $95 \%$ confidence level, are used to correct the background fractions. Thus, each correction causes a change in the estimated contamination. The branching ratio results are affected by less than 0.001 . The effect of variations in the assumed Monte Carlo branching ratios is also negligible.

The results from this analysis are consistent with the values $B(\tau \rightarrow \mathrm{e} \nu \bar{\nu})=(17.4 \pm 0.5 \pm$ $0.4) \%$ and $B(\tau \rightarrow \mu \nu \bar{\nu})=(16.8 \pm 0.5 \pm 0.4) \%$ based on the 1990 OPAL data [1]. We have followed the procedure of [12] to combine these results and produce a measurement based on the OPAL data recorded between 1990 and 1992. The correlation coefficients of the errors between these results are 0.25 and 0.03 for the $\tau \rightarrow$ e $\nu \bar{\nu}$ and $\tau \rightarrow \mu \nu \bar{\nu}$ channels respectively. The final results are

$$
\begin{aligned}
& B(\tau \rightarrow \mathrm{e} \nu \bar{\nu})=(18.04 \pm 0.33) \% \\
& B(\tau \rightarrow \mu \nu \bar{\nu})=(17.36 \pm 0.27) \%
\end{aligned}
$$

These branching ratio results are consistent with the published results in both leptonic channels. The most precise $B(\tau \rightarrow$ e $\nu \bar{\nu})$ result is $(17.97 \pm 0.27) \%$ from the CLEO experiment [13] and the Particle Data Group (1994) average value is $(17.90 \pm 0.17) \%$ [14]. For $B(\tau \rightarrow \mu \nu \bar{\nu})$ the Particle Data Group (1994) average value is $(17.44 \pm 0.23) \%$ [14] and the most precise published result to date is $(17.35 \pm 0.55) \%$ from the ALEPH experiment [15].

\section{Charged Current Universality tests}

The measured leptonic branching ratios of the tau can be used to make a direct test of muonelectron universality. The leptonic width in tau decay is given by [16]:

$$
\Gamma(L \rightarrow l \nu \bar{\nu})=\frac{g_{L}^{2} g_{l}^{2}}{\left(8 M_{W}^{2}\right)^{2}} \frac{M_{L}^{5}}{96 \pi^{3}} f\left(\frac{M_{l}^{2}}{M_{L}^{2}}\right)\left(1+\frac{3 M_{L}^{2}}{5 M_{W}^{2}}\right)\left[1+\frac{\alpha\left(M_{L}\right)}{2 \pi}\left(\frac{25}{4}-\pi^{2}\right)\right],
$$

where $g_{L}$ and $g_{l}$ are the electroweak couplings of the parent and daughter leptons, $M_{L}$ and $M_{l}$ their masses, $M_{W}$ the $\mathrm{W}$ boson mass and $\alpha\left(M_{L}\right)$ is the fine structure constant evaluated at the mass of lepton L. At the mass of the $\tau$ the fine structure constant is $\frac{1}{133.29}$ [17]. The function $f(x)$ is a factor which takes into account the mass of the final state lepton, and is defined as

$$
f(\mathrm{x})=1-8 \mathrm{x}+8 \mathrm{x}^{3}-\mathrm{x}^{4}-12 \mathrm{x}^{2} \ln (\mathrm{x}) .
$$

Assuming the neutrino to be massless and using the current world average tau mass of $\left(1777.1{ }_{-0.5}^{+0.4}\right) \mathrm{MeV}$ [14], this function has values of 1.0000 and 0.9726 for the electron and muon respectively. The last two terms in the brackets correspond to electroweak and photonic radiative corrections which are not included into the effective couplings [17]. The numerical values of these factors are 1.0003 and 0.9957 respectively.

The ratio of widths for tau decay into muons and electrons, which equals the ratio of the respective leptonic branching ratios, is related to $g_{\mu}$ and $g_{e}$ via:

$$
\frac{\Gamma(\tau \rightarrow \mu \nu \bar{\nu})}{\Gamma(\tau \rightarrow \mathrm{e} \nu \bar{\nu})}=\frac{g_{\mu}^{2}}{g_{e}^{2}}\left[\frac{f\left(\frac{M_{\mu}^{2}}{M_{\tau}^{2}}\right)}{f\left(\frac{M_{e}^{2}}{M_{\tau}^{2}}\right)}\right]
$$


Using the tau leptonic branching fractions measured in this paper, this yields

$$
\frac{g_{\mu}}{g_{e}}=0.994 \pm 0.012
$$

where the statistical and systematic errors have been added in quadrature. This result supports the hypothesis of charged current electron-muon universality. The most precise tests of this universality have been made by measuring the pion leptonic branching ratios [18]. Assuming this universality the branching ratio results for $\tau \rightarrow \mathrm{e} \nu \bar{\nu}$ and $\tau \rightarrow \mu \nu \bar{\nu}$ can be combined, after correcting for the difference in electron and muon masses, to produce a combined measurement of the $\tau$ electronic branching ratio of $\bar{B}_{\mathrm{e}}=(17.93 \pm 0.21) \%$.

A separate universality test can be made by comparing the partial widths for the decays of the tau and muon into electrons. The electronic partial width for muon decay is given by the above formula where $\mathrm{L}=\mu$ and $1=\mathrm{e}$. By taking the ratio with the corresponding tau width this results in

$$
\frac{\Gamma(\tau \rightarrow \mathrm{e} \nu \bar{\nu})}{\Gamma(\mu \rightarrow e \nu \bar{\nu})}=\frac{g_{\tau}^{2}}{g_{\mu}^{2}} \frac{M_{\tau}^{5}}{M_{\mu}^{5}} 1.0004 .
$$

This can be written in terms of the electronic branching ratios of the tau and muon and their lifetimes, to obtain an expression relating $g_{\tau}$ and $g_{\mu}$ :

$$
\frac{g_{\tau}^{2}}{g_{\mu}^{2}}=\frac{\tau_{\mu}}{\tau_{\tau}} B(\tau \rightarrow \mathrm{e} \nu \bar{\nu})\left[\frac{\mathrm{M}_{\mu}}{\mathrm{M}_{\tau}}\right]^{5} 0.9996
$$

Substituting the muon lifetime and the tau and muon masses [14], the OPAL measurement of the tau lifetime $288.8 \pm 2.2 \pm 1.4$ fsecs [19] and the electronic branching ratio of the tau measured in this analysis, this gives

$$
\frac{g_{\tau}}{g_{\mu}}=1.010 \pm 0.010
$$

The measured ratio $g_{\mu} / g_{e}$ and the muonic branching ratio of the tau can be used to extract the ratio $g_{\tau} / g_{e}$ as follows:

$$
\frac{g_{\tau}^{2}}{g_{e}^{2}}=\frac{\tau_{\mu}}{\tau_{\tau}} B(\tau \rightarrow \mu \nu \bar{\nu})\left[\frac{M_{\mu}}{M_{\tau}}\right]^{5}(1.028)
$$

From this expression

$$
\frac{g_{\tau}}{g_{e}}=1.005 \pm 0.009
$$

Again, the branching ratio errors (statistical and systematic) have been combined in quadrature with the lifetime uncertainty to estimate the final error. The errors in $g_{\tau} / g_{\mu}$ and $g_{\tau} / g_{e}$ are calculated from the uncertainties in the mass, lifetime and branching ratios of the tau. The contribution from the various masses including the tau is negligible and the current error contribution from the branching ratio is slightly larger than that from the lifetime. Under the assumption of electron-muon universality, the ratio $g_{\tau} / g_{e \mu}=1.007 \pm 0.007$ is measured in this experiment. 
The tau lifetime and the averaged tau electronic branching ratio from the OPAL experiment are displayed in Figure 8. Also shown in this figure is the line representing the standard model relationship between these quantities which is calculated assuming lepton universality. The small width of the line reflects the high precision of the tau mass measurement since theoretical uncertainties on the prediction are negligible.

All of the measured ratios of electroweak leptonic coupling constants are consistent with unity. We conclude that the OPAL branching ratio results and tau lifetime measurements support the hypothesis of lepton universality.

\section{Determination of $\alpha_{s}$ from Tau Decay}

The strong coupling $\alpha_{s}$ can be extracted from the ratio $R_{\tau}=B\left(\tau \rightarrow\right.$ hadrons $\left.+\nu_{\tau}\right) / B(\tau \rightarrow$ $\left.\mathrm{e} \bar{\nu}_{\mathrm{e}} \nu_{\tau}\right)[20] . R_{\tau}$ can be written as $\left(1-\bar{B}_{\mathrm{e}}(1+0.9726)\right) / \bar{B}_{\mathrm{e}}$ where $\bar{B}_{\mathrm{e}}$ is defined in the previous section. The branching ratio $\bar{B}_{\mathrm{e}}$ can also be independently determined from measurements of the lifetime of the $\tau$ via the relation $\bar{B}_{\mathrm{e}}=\left(\tau_{\tau} / \tau_{\mu}\right)\left(M_{\tau} / M_{\mu}\right)^{5}$, where the masses and muon lifetime are taken from [14].

The experimental value of $R_{\tau}$, obtained from the $B(\tau \rightarrow$ e $\nu \bar{\nu})$ and $B(\tau \rightarrow \mu \nu \bar{\nu})$ measurements using 1990-1992 data presented in this paper, is $3.605 \pm 0.064$. The $R_{\tau}$ value calculated from the OPAL measurement of the tau lifetime using 1990-1993 data [19] is $3.682 \pm 0.048$. These two experimental $R_{\tau}$ values are combined to yield $3.654 \pm 0.038$. The $\alpha_{s}$ value was extracted using the technique described in [21]. The resulting $\alpha_{s}$ value from the OPAL $R_{\tau}$ measurement is $0.375_{-0.018-0.017-0.006}^{+0.019+0.025+006}$ at $Q^{2}=M_{\tau}^{2}$, based on the $\mathcal{O}\left(\alpha_{s}^{3}\right)$ theoretical prediction with three quark flavours, where the first error is the combined experimental error, the second is due to higher order effects and the third is due to uncertainties in the non-perturbative contribution. In order to estimate the uncertainties from higher order QCD effects we apply the following method which follows the strategy used in previous $\alpha_{s}$ studies [22, 23]. Three effects are considered: varying the renormalisation scale from $1.0 \mathrm{GeV}$ to $2.5 \mathrm{GeV}$; introducing a fourth order coefficient in the integrand of the spectral function; and introducing a fourth order coefficient in the renormalisation group equation of $\alpha_{s}$. These three error estimates are then combined by adding them in quadrature. We remark that it has been controversially argued [24] that the presence of hadronic resonances limits the applicability of perturbative QCD in $\tau$ decays and that consequentially the theoretical errors may be underestimated. The error estimate from non-perturbative effects is taken from [20] and [21] which is based on the Shifman, Vainshtein and Zakharov (SVZ) parameterisation [25]. In the SVZ parameterisation the so called 'dimension $D=2$ term' is zero. We note that there is some controversy regarding the limit on the magnitude of a possible $D=2$ term $[26,27,28,29]$ which potentially introduces a systematic uncertainty in addition to those quoted above. In particular, reference [28] quotes the possible error on $\alpha_{s}$ at $Q^{2}=M_{\tau}^{2}$ from this effect as 0.02 while reference [27] gives the range 0.05 to 0.08 for this error.

The $\alpha_{s}$ value at $Q^{2}=M_{Z}^{2}$ is calculated to be $0.1229_{-0.0017}^{+0.0016+0.0025}$ after extrapolation from $Q^{2}=M_{\tau}^{2}$ using the renormalisation group equation [30], where the first error is the experimental error from the branching fractions and lifetime measurements and the second is the quadratic sum of contributions arising from various theoretical uncertainties. The theoretical error of 
${ }_{-0.0021}^{+0.0025}$ includes a contribution of \pm 0.0011 from the ambiguity of the charm and bottom threshold

effects in the extrapolation [22]; ${ }_{-0.0017}^{+0.0022}$ from higher order perturbative QCD effects and \pm 0.0005 from uncertainties in the non-perturbative contribution based on the SVZ parameterisation. A potential additional systematic error in $\alpha_{s}\left(Q^{2}=M_{\mathrm{Z}}^{2}\right)$ due to the possible $D=2$ contribution beyond the SVZ parameterisation also exists as discussed above. This is estimated to be as much as $\pm 0.002[28]$ or \pm 0.005 [29]. The measured value of $\alpha_{s}$ is in good agreement with the value of $0.126 \pm 0.005 \pm 0.002$, where the first error is statistical and the second systematic, obtained from the $\mathrm{Z}^{0}$ line shape analysis [31] and also with results obtained from hadronic event shape distributions in (resummed) $\mathcal{O}\left(\alpha_{s}^{2}\right)[22]$.

\section{Conclusions}

The tau leptonic branching ratios extracted from the 1990, 1991 and 1992 data samples recorded by the OPAL detector are:

$$
\begin{aligned}
& B(\tau \rightarrow \mathrm{e} \nu \bar{\nu})=(18.04 \pm 0.33) \% \\
& B(\tau \rightarrow \mu \nu \bar{\nu})=(17.36 \pm 0.27) \%
\end{aligned}
$$

These results are consistent with the Particle Data Group (94) averages of $(17.90 \pm 0.17) \%$, and $(17.44 \pm 0.23) \%[14]$ for $\tau \rightarrow \mathrm{e} \nu \bar{\nu}$ and $\tau \rightarrow \mu \nu \bar{\nu}$ respectively and with other LEP measurements from the ALEPH [15], DELPHI [32] and L3 [33] collaborations.

The extracted leptonic branching ratios of the tau have been used together with other properties of the tau and muon to test the universality of the leptonic charged-current couplings. Current branching ratio measurements from OPAL show consistency with this hypothesis at the $1 \%$ level.

The ratio $R_{\tau}=B\left(\tau \rightarrow\right.$ hadrons $\left.+\nu_{\tau}\right) / B\left(\tau \rightarrow \mathrm{e} \bar{\nu}_{\mathrm{e}} \nu_{\tau}\right)$ is calculated from the leptonic branching fractions of tau decay as well as from the tau lifetime measured by the OPAL collaboration. The $\alpha_{s}$ value at $Q^{2}=M_{\tau}^{2}$ is extracted from the $R_{\tau}$ value assuming the parameterisation of the non-perturbative effects as proposed by Shifman, Vainstein and Zakharov. The $\alpha_{s}$ value, which is extrapolated from $Q^{2}=M_{\tau}^{2}$ to $Q^{2}=M_{Z}^{2}$ by using the renormalisation group equation, is $0.1229_{-0.0017}^{+0.0016}$ (experiment $)_{-0.0021}^{+0.0025}$ (theory) with a possible additional uncertainty of as much as $\pm 0.002[28]$ or \pm 0.005 [29] from effects beyond the SVZ parametrisation. This value of $\alpha_{s}$ is in good agreement with the value obtained from the $Z^{0}$ line shape analysis.

\section{Acknowledgements:}

We thank F. Le Diberder for the use of his programmes in the extraction of $\alpha_{s}$. It is a pleasure to thank the SL Division for the efficient operation of the LEP accelerator, the precise information on the absolute energy, and their continuing close cooperation with our experimental group. In addition to the support staff at our own institutions we are pleased to acknowledge the 
Department of Energy, USA,

National Science Foundation, USA,

Particle Physics and Astronomy Research Council, UK,

Natural Sciences and Engineering Research Council, Canada,

Fussefeld Foundation,

Israel Ministry of Science,

Israel Science Foundation, administered by the Israel Academy of Science and Humanities, Minerva Gesellschaft,

Japanese Ministry of Education, Science and Culture (the Monbusho) and a grant under the Monbusho International Science Research Program,

German Israeli Bi-national Science Foundation (GIF),

Direction des Sciences de la Matière du Commissariat à l'Energie Atomique, France,

Bundesministerium für Forschung und Technologie, Germany,

National Research Council of Canada,

A.P. Sloan Foundation and Junta Nacional de Investigação Científica e Tecnológica, Portugal. 


\section{References}

[1] The OPAL Collaboration, G. Alexander et al., Phys. Lett. B266 (1991) 201.

[2] The OPAL Collaboration, K. Ahmet et al., Nucl. Instr. and Meth., A305 (1991) 275.

[3] P. P. Allport et al., Nucl. Instr. and Meth., A324 (1993) 34.

[4] J. Allison et al., Comp. Phys. Comm. 47 (1987) 55.

R. Brun et al., GEANT 3, Report DD/EE/84-1, CERN (1989).

D. R. Ward, in Proceedings of the MC'91 Workshop, NIKHEF, Amsterdam, 1991.

[5] M. Arignon et al., Nucl. Instr. and Meth., A313 (1992) 103.

[6] D. G. Charlton, F. Meijers, T. J. Smith, P. S. Wells, Nucl. Instr. and Meth., A325 (1993) 129.

[7] M. Böhm, A. Denner and W. Hollik, Nucl. Phys. B304 (1988) 687;

F. A. Berends, R. Kleiss, W. Hollik, Nucl. Phys. B304 (1988) 712; (BABAMC).

[8] S. Jadach, B. F. L. Ward, and Z. Was, Comp. Phys. Comm. 66 (1991) 276; (KORALZ, Version 3.8).

[9] T. Sjöstrand, Comp. Phys. Comm. 39 (1986) 347; (JETSET, Version 7.1).

[10] R. Bhattacharya, J. Smith, G. Grammer, Phys. Rev. D15 (1977) 3267;

J. Smith, J. A. M. Vermaseren, G. Grammer, Phys. Rev. D15 (1977) 3280.

[11] S. Jadach, J. H. Kühn, and Z. Was, Comp. Phys. Comm. 64 (1991) 275; (TAUOLA, Version 1.5).

[12] L. Lyons, D. Gibaut and P. Clifford, Nucl. Instr. and Meth. A270 (1988) 110 .

[13] The CLEO collaboration, O. S. Akerib et al., Phys. Rev. Lett. 69 (1992) 3610.

[14] 'Review of Particle Properties', L. Montanet et al., Phys. Rev. D50 (1994) 1173.

[15] The ALEPH collaboration, D. Decamp et al., Z. Phys. C54 (1992) 211.

[16] W. J. Marciano, Ann. Rev. Nucl. Part. Sci. 41 (1991) 469.

[17] W. J. Marciano and A. Sirlin, Phys. Rev. Lett. 61 (1988) 1815.

[18] D. Britton et al., Phys. Rev. Lett. 68 (1992) 3000.

G. Czapek et al., Phys. Rev. Lett. 70 (1993) 17.

[19] The OPAL Collaboration, R. Akers et al., Phys. Lett. B338 (1994) 497.

[20] E. Braaten, S. Narison and A. Pich, Nucl. Phys. B373 (1992) 581 and references therein.

[21] F. Le Diberder and A. Pich, Phys. Lett. 286B (1992) 147.

[22] The OPAL collaboration, P. Acton et al., Z. Phys. C55 (1992) 1. 
[23] The ALEPH Collaboration, D. Buskulic et al., Phys. Lett. B307 (1993) 209.

[24] T. N. Truong, Phys. Rev. D47, (1993) 3999.

[25] M. A. Shifman, A. L. Vainstein and V. I. Zakharov, Nucl. Phys. B147 (1979) 385,448,519.

[26] S. Narison, Phys. Lett. B300 (1993) 293.

[27] G. Altarelli, Proceedings of the Third Workshop on Tau Lepton Physics, Montreux, (1994), CERN-TH 7493 (1994)

[28] S. Narison, Proceedings of the Third Workshop on Tau Lepton Physics, Montreux, (1994), CERN-TH 7506 (1994)

[29] G. Altarelli, P. Nason and G. Ridolphi, CERN-TH 7537 (1994)

[30] W. Bernreuther and W. Wetzel, Nucl. Phys. B197 (1982) 228.

[31] The LEP Collaborations, ALEPH DELPHI L3 OPAL, and LEP Electroweak working group, CERN-PPE 94-187 (1994)

[32] The DELPHI collaboration, P. Abreu et al. , Z. Phys. C55 (1992) 555.

[33] The L3 collaboration, O. Adriani et al. Phys. Lett. B236 (1993) 1. 


\begin{tabular}{|l||c|c|}
\hline Background & $\begin{array}{c}\text { Monte Carlo Predicted } \\
\text { Contamination(\%) }\end{array}$ & $\begin{array}{c}\text { Corrected } \\
\text { Contamination }(\%)\end{array}$ \\
\hline \multicolumn{3}{|c|}{$\tau$ pairs } \\
\hline $\mathrm{e}^{+} \mathrm{e}^{-} \rightarrow \mathrm{e}^{+} \mathrm{e}^{-}$ & $0.13 \pm 0.03$ & $0.24 \pm 0.07$ \\
$\mathrm{e}^{+} \mathrm{e}^{-} \rightarrow \mu^{+} \mu^{-}$ & $0.45 \pm 0.02$ & $1.00 \pm 0.28$ \\
$\mathrm{e}^{+} \mathrm{e}^{-} \rightarrow q \bar{q}$ & $0.42 \pm 0.05$ & $0.42 \pm 0.08$ \\
$\mathrm{e}^{+} \mathrm{e}^{-} \rightarrow\left(\mathrm{e}^{+} \mathrm{e}^{-}\right) \mathrm{e}^{+} \mathrm{e}^{-}$ & $0.09 \pm 0.02$ & $0.09 \pm 0.02$ \\
$\mathrm{e}^{+} \mathrm{e}^{-} \rightarrow\left(\mathrm{e}^{+} \mathrm{e}^{-}\right) \mu^{+} \mu^{-}$ & $0.07 \pm 0.02$ & $0.08 \pm 0.02$ \\
\hline Total & $1.17 \pm 0.06$ & $1.83 \pm 0.30$ \\
\hline \hline \multicolumn{2}{|c|}{$\tau \rightarrow \mathrm{e} \nu \bar{\nu}$} \\
\hline \hline$\tau \rightarrow \pi(\mathrm{K}) \nu$ & $3.99 \pm 0.09$ & $2.51 \pm 0.74$ \\
$\tau \rightarrow \rho \nu$ & $1.67 \pm 0.06$ & $1.67 \pm 0.29$ \\
$\tau \rightarrow$ other & $0.34 \pm 0.03$ & $0.34 \pm 0.08$ \\
\hline $\mathrm{e}^{+} \mathrm{e}^{-} \rightarrow \mathrm{e}^{+} \mathrm{e}^{-}$ & $0.13 \pm 0.05$ & $0.13 \pm 0.06$ \\
$\mathrm{e}^{+} \mathrm{e}^{-} \rightarrow \mu^{+} \mu^{-}$ & $0.04 \pm 0.01$ & $0.04 \pm 0.03$ \\
$\mathrm{e}^{+} \mathrm{e}^{-} \rightarrow\left(\mathrm{e}^{+} \mathrm{e}^{-}\right) \mathrm{e}^{+} \mathrm{e}^{-}$ & $0.41 \pm 0.09$ & $0.41 \pm 0.09$ \\
\hline Total & $6.59 \pm 0.13$ & $5.11 \pm 0.81$ \\
\hline \hline \multicolumn{2}{|c||c|}{$\tau \rightarrow \bar{\nu}$} \\
\hline \hline$\tau \rightarrow \pi(\mathrm{K}) \nu$ & $2.23 \pm 0.07$ & $1.32 \pm 0.46$ \\
$\tau \rightarrow$ other & $0.07 \pm 0.01$ & $0.07 \pm 0.04$ \\
\hline $\mathrm{e}^{+} \mathrm{e}^{-} \rightarrow \mu^{+} \mu^{-}$ & $0.22 \pm 0.03$ & $0.22 \pm 0.12$ \\
$\mathrm{e}^{+} \mathrm{e}^{-} \rightarrow\left(\mathrm{e}^{+} \mathrm{e}^{-}\right) \mu^{+} \mu^{-}$ & $0.37 \pm 0.06$ & $0.28 \pm 0.06$ \\
\hline Total & $2.90 \pm 0.09$ & $1.90 \pm 0.48$ \\
\hline
\end{tabular}

Table 1: Estimated background fractions before and after applying corrections using systematic studies discussed in the text. 


\begin{tabular}{|l|c|c|}
\hline & $\tau \rightarrow \mathrm{e} \nu \bar{\nu}$ & $\tau \rightarrow \mu \nu \bar{\nu}$ \\
\hline Total uncorrected efficiency (\%) & $72.7 \pm 0.2$ & $85.7 \pm 0.2$ \\
Correction factor & $0.981 \pm 0.007 \pm 0.009$ & $0.980 \pm 0.009 \pm 0.006$ \\
Total corrected efficiency $(\%)$ & $71.3 \pm 0.9$ & $84.0 \pm 0.9$ \\
\hline
\end{tabular}

Table 2: Correction factors and selection efficiencies (\%) for the selection of $\tau \rightarrow$ e $\nu \bar{\nu}$ and $\tau \rightarrow \mu \nu \bar{\nu}$.

\begin{tabular}{|c|c|c|}
\hline $\begin{array}{l}N_{C \text { Cand }}^{\tau} \\
f_{B k g d}^{\tau}(\%)\end{array}$ & \multicolumn{2}{|c|}{$\begin{array}{c}54392 \\
1.83 \pm 0.30\end{array}$} \\
\hline & $\tau \rightarrow \mathrm{e} \nu \bar{\nu}$ & $\tau \rightarrow \mu \nu \bar{\nu}$ \\
\hline$N_{\text {Cand }}^{\tau \rightarrow \ell \nu \bar{\nu}}$ & 7322 & 7941 \\
\hline$f_{B k g d}^{\tau \rightarrow \ell \nu \bar{\nu}}(\%)$ & $5.11 \pm 0.81$ & $1.90 \pm 0.48$ \\
\hline$\epsilon^{\tau \rightarrow \ell \nu \bar{\nu}}(\%)$ & $71.3 \pm 0.9$ & $84.0 \pm 0.9$ \\
\hline$F_{B i a s}^{\tau \rightarrow \ell \nu \bar{\nu}}$ & $1.006 \pm 0.004$ & $0.994 \pm 0.004$ \\
\hline 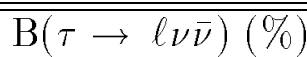 & $\overline{18.14 \pm 0.20 \pm 0.28}$ & $=17.48 \pm 0.18 \pm 0.23$ \\
\hline
\end{tabular}

Table 3: Background fractions, corrected efficiencies, selection biases and final branching ratio measurements for selected $\tau \rightarrow \mathrm{e} \nu \bar{\nu}$ and $\tau \rightarrow \mu \nu \bar{\nu}$ candidate events using the complete 1991 and 1992 data samples

\begin{tabular}{|l|c|c|}
\hline \multicolumn{3}{|c|}{ Contributions to the Systematic Error $(\%)$} \\
\hline & $\tau \rightarrow \mathrm{e} \nu \bar{\nu}$ & $\tau \rightarrow \mu \nu \bar{\nu}$ \\
\hline$f_{B k g d}^{\tau}$ & 0.06 & 0.05 \\
$f_{B k \ell d}^{\tau \rightarrow \ell}$ & 0.15 & 0.09 \\
$\epsilon^{\tau \rightarrow \ell \nu \bar{\nu}}$ & 0.22 & 0.20 \\
$F_{\text {Bias }}^{\tau \rightarrow \ell \nu \bar{\nu}}$ & 0.07 & 0.07 \\
\hline Total & 0.28 & 0.23 \\
\hline
\end{tabular}

Table 4: Breakdown of the contributions to the absolute systematic errors on the branching ratios for $\tau \rightarrow \mathrm{e} \nu \bar{\nu}$ and $\tau \rightarrow \mu \nu \bar{\nu}$, quoted in Table 3, using the complete 1991 and 1992 data samples. 

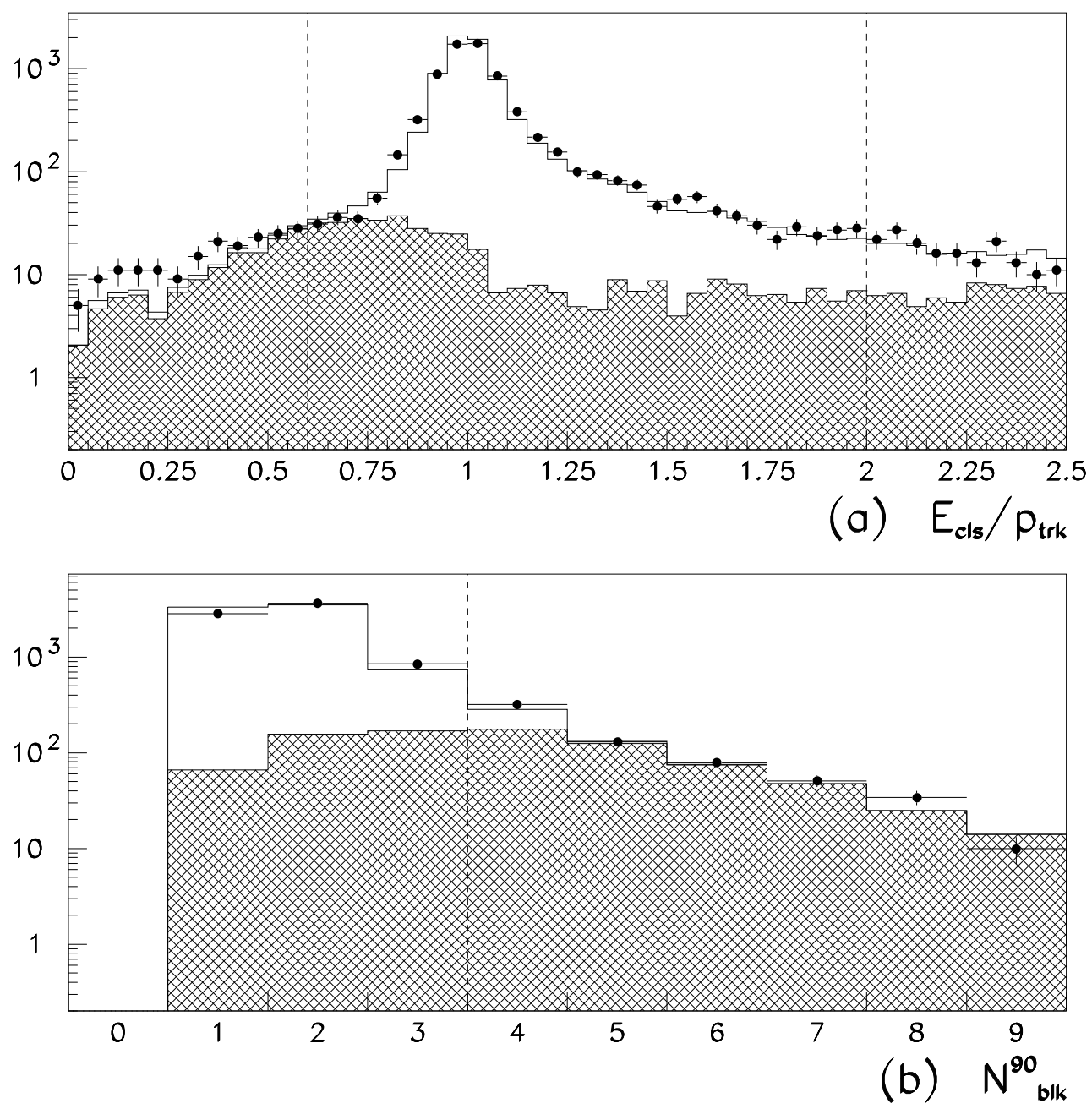

Figure 1: Plots of the distributions of some of the variables used in the $\tau \rightarrow$ ev $\bar{\nu}$ selection. In this and the following figures the data are represented as points and the Monte Carlo expectations as histograms. The expected backgrounds are shown as hatched histograms and have been scaled by the correction factors discussed in section 4.1. The values at which cuts are imposed are shown as dashed vertical lines. The figures show: a) associated cluster energy divided by the track momentum; and b) the number of lead-glass blocks containing at least $90 \%$ of the cluster energy for the cluster associated to the track. 

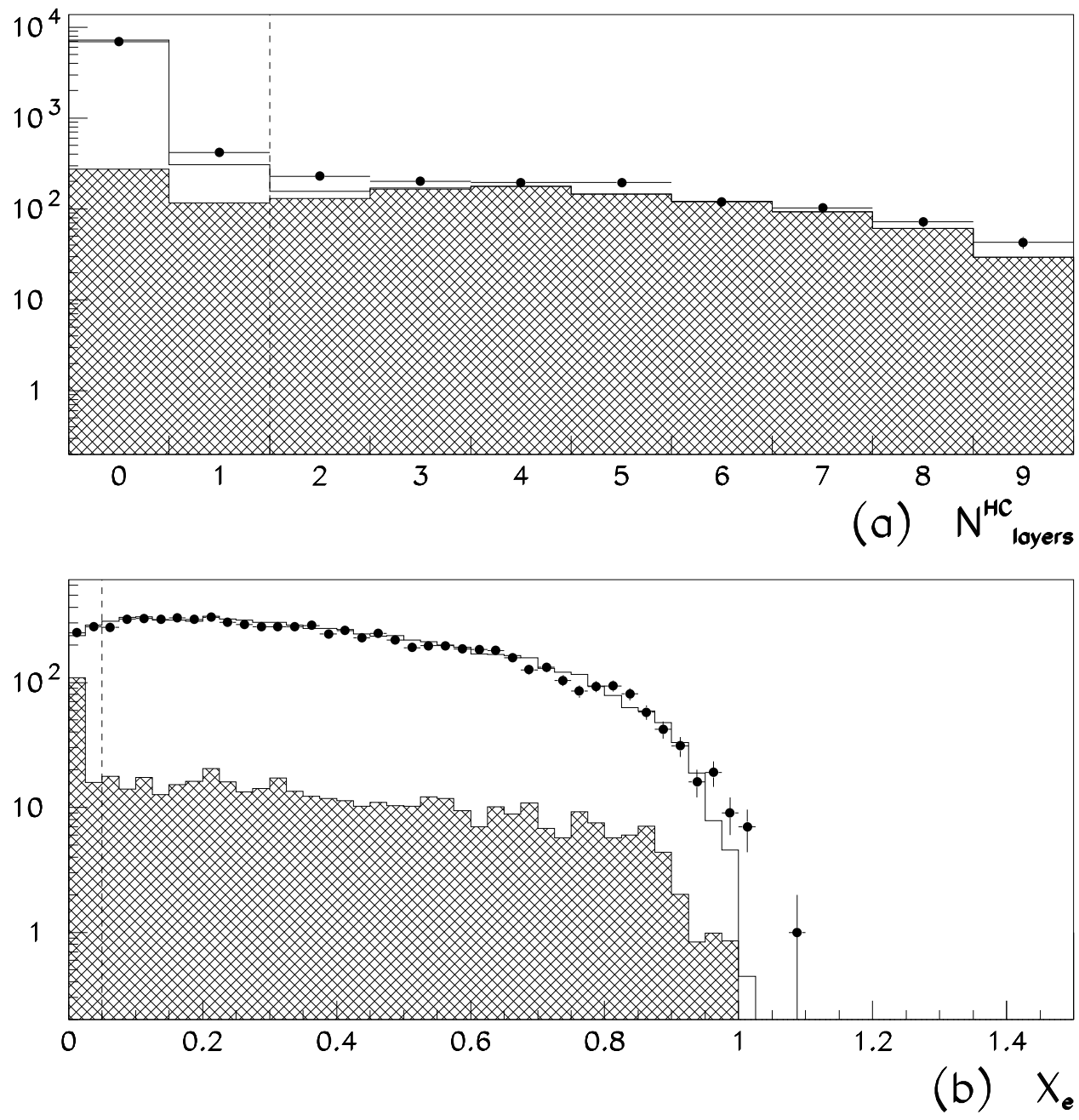

Figure 2: Further distributions of variables used in the $\tau \rightarrow$ e $\nu \bar{\nu}$ selection: a) the number of HCAL layers in the cone; and b) the total shower energy in the cone divided by the beam energy. 

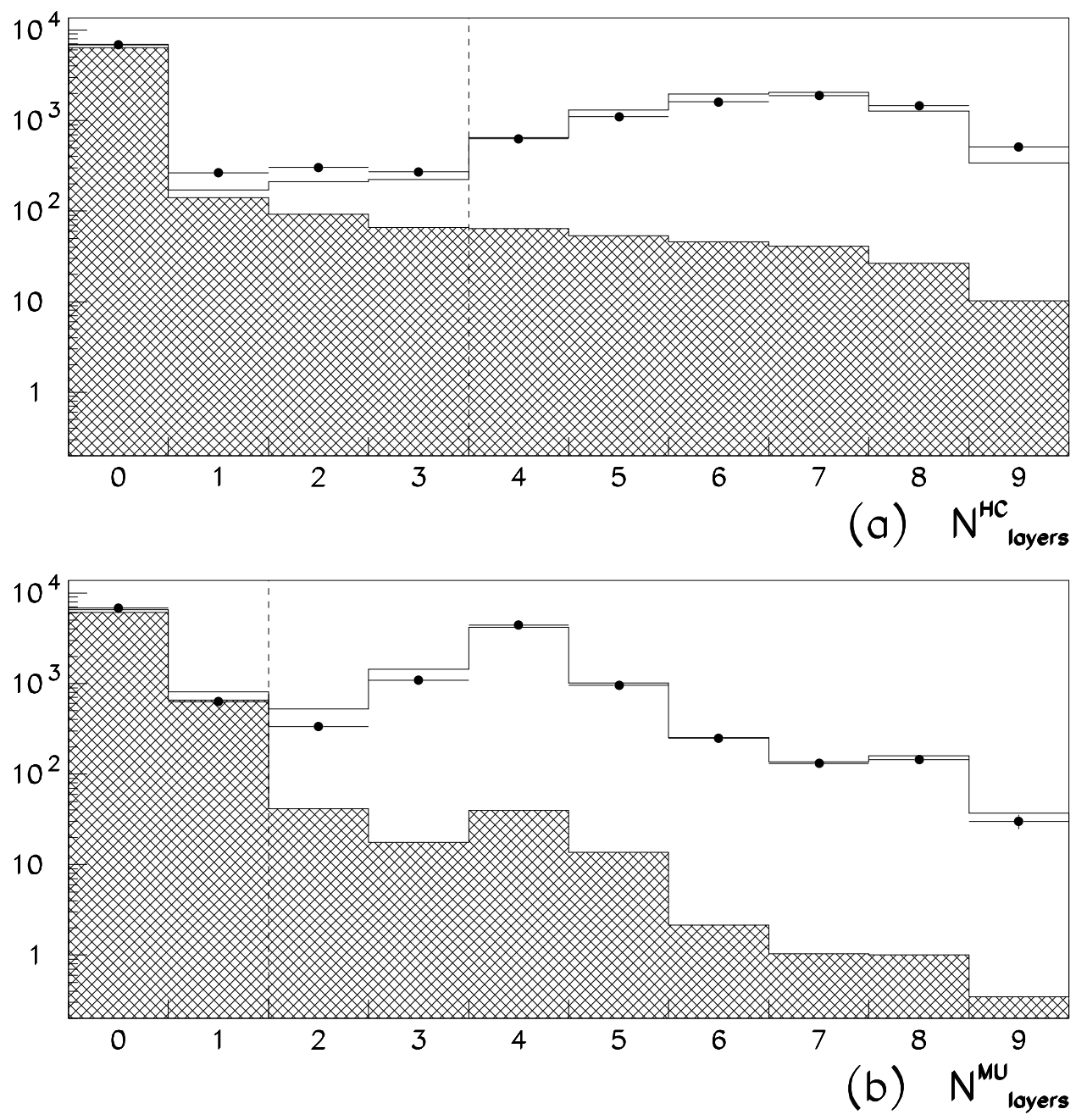

Figure 3: Distributions of some of the variables used in the $\tau \rightarrow \mu \nu \bar{\nu}$ selection: a) the number of layers of HCAL with hits associated to the candidate track; and b) the number of MUON chamber layers containing hits associated to the candidate track. 

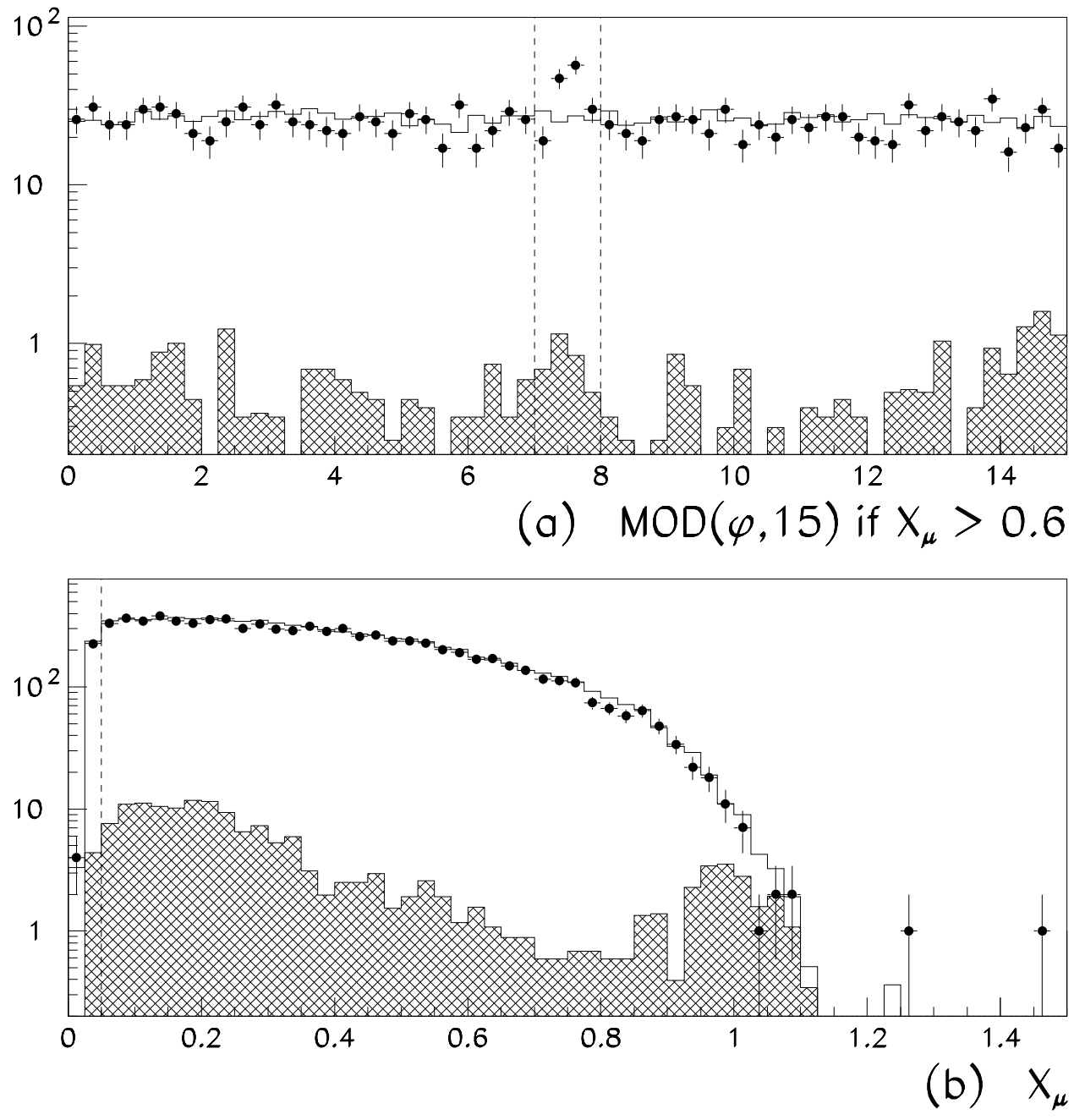

Figure 4: Further distributions of variables used in the $\tau \rightarrow \mu \nu \bar{\nu}$ selection: a) $\bmod \left(\phi, 15^{\circ}\right)$, the azimuthal angle of the track, measured relative to the jet chamber sector through which it passes (the cathode planes lie at $0^{\circ}$ and $15^{\circ}$ while the anode planes are at $7.5^{\circ}$ ); and $b$ ) the distribution of the variable $x_{\mu}$, defined in section 3.3, after all other cuts have been applied. 

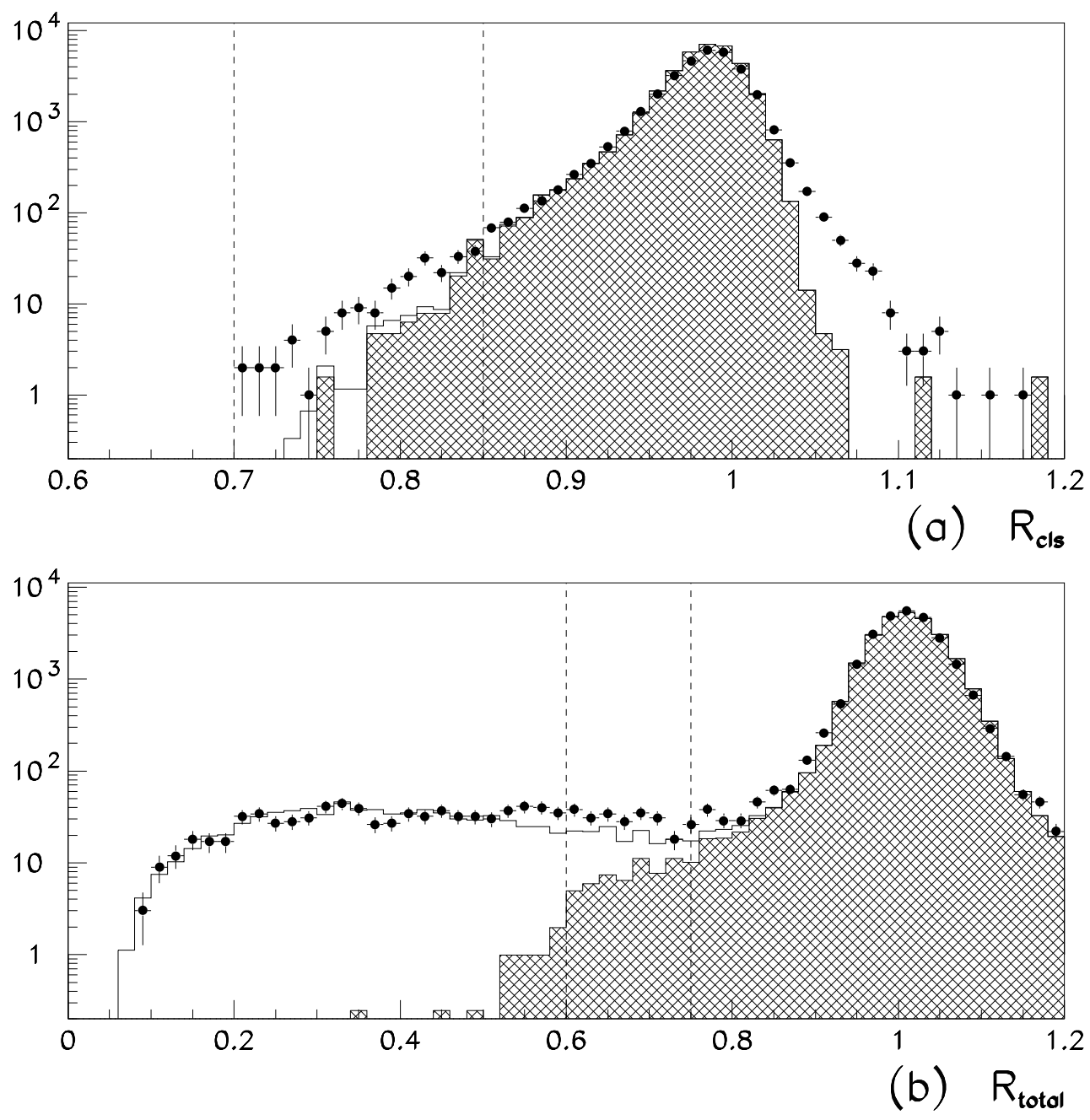

Figure 5: Distributions of variables used in selecting control samples for background studies. The data are represented as points, the Monte Carlo expectations as histograms with the background under study as hatched histogram: a) the total energy deposited in the lead glass calorimeter divided by the centre of mass energy; and b) the scalar sum of the highest energy cluster and the highest momentum track of both cones divided by $\mathrm{E}_{\mathrm{cm}}$. 

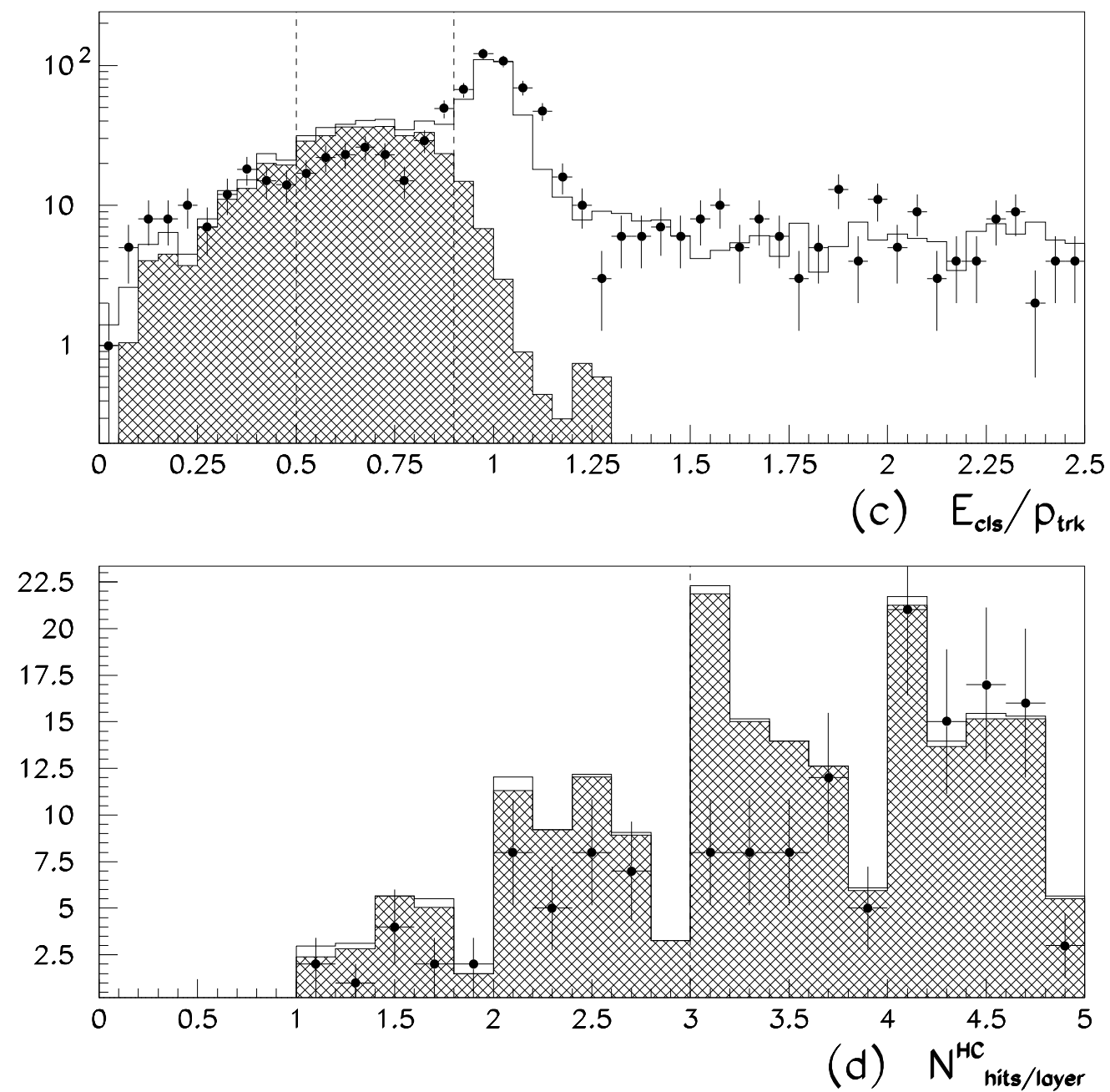

Figure 6: Further distributions of variables used in selecting control samples for background studies. The data are represented as points, the Monte Carlo expectations as histograms with the background under study as hatched histogram: a) the ratio of the energy of the cluster associated to the electron candidate and the candidate track's reconstructed momentum, $\mathrm{E}_{\mathrm{cls}} / \mathrm{p}_{\text {trk }}$; and $\mathrm{b}$ ) the mean number of hit strips which have been associated with a charged track, averaged over all the planes of the HCAL which show activity. 

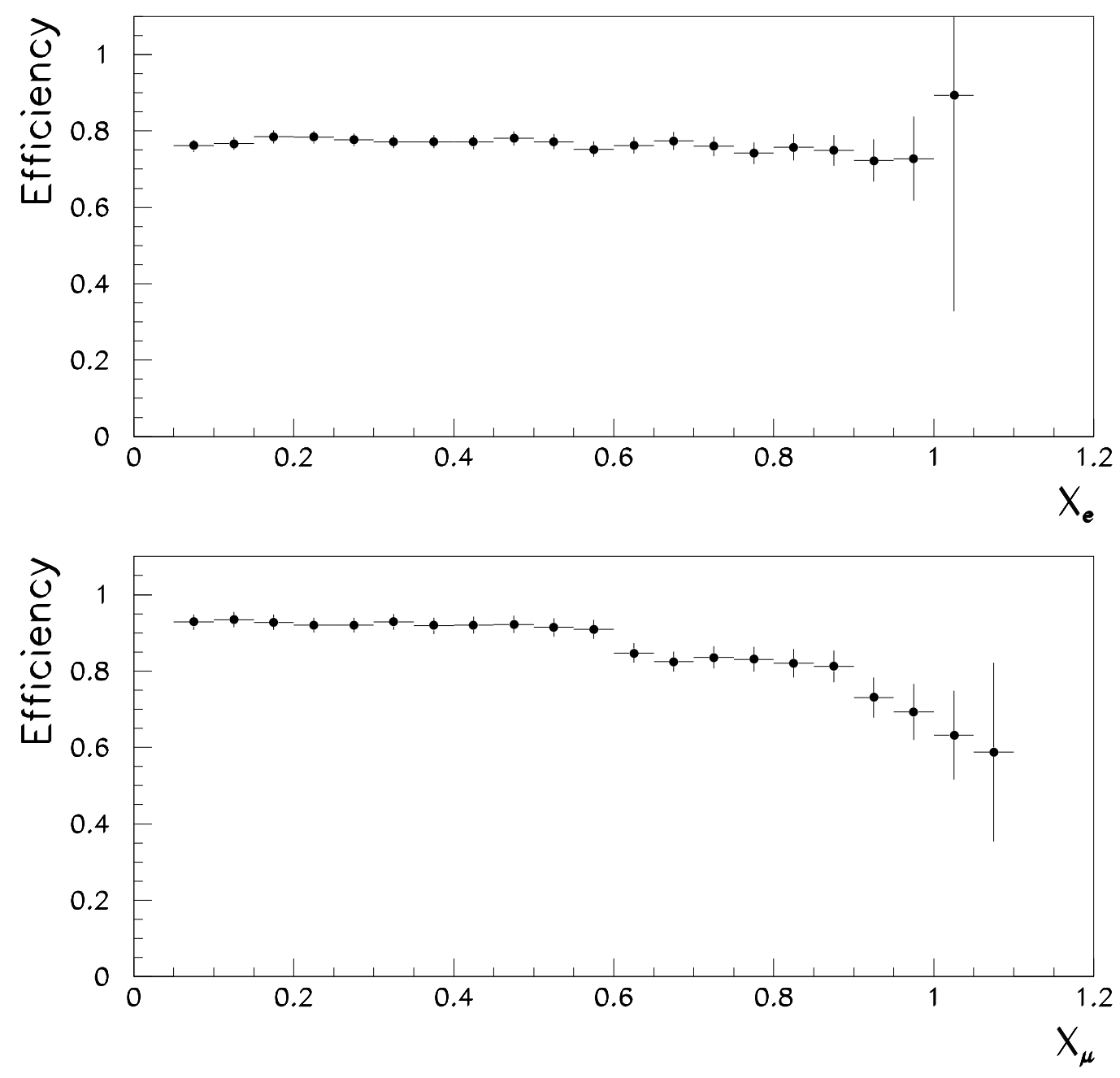

Figure 7: The efficiencies determined from the Monte Carlo for identifying from the barrel $\tau$ pair sample a) the decay $\tau \rightarrow \mathrm{e} \nu \bar{\nu}$ as a function of the reconstructed $\mathrm{x}_{\mathrm{e}}$; and $\mathrm{b}$ ) the decay $\tau \rightarrow \mu \nu \bar{\nu}$ as a function of the reconstructed $\mathrm{x}_{\mu}$. The selection cuts for this decay change at $\mathrm{x}_{\mu}$ values of 0.6 and 0.9 as described in section 3.3 . 


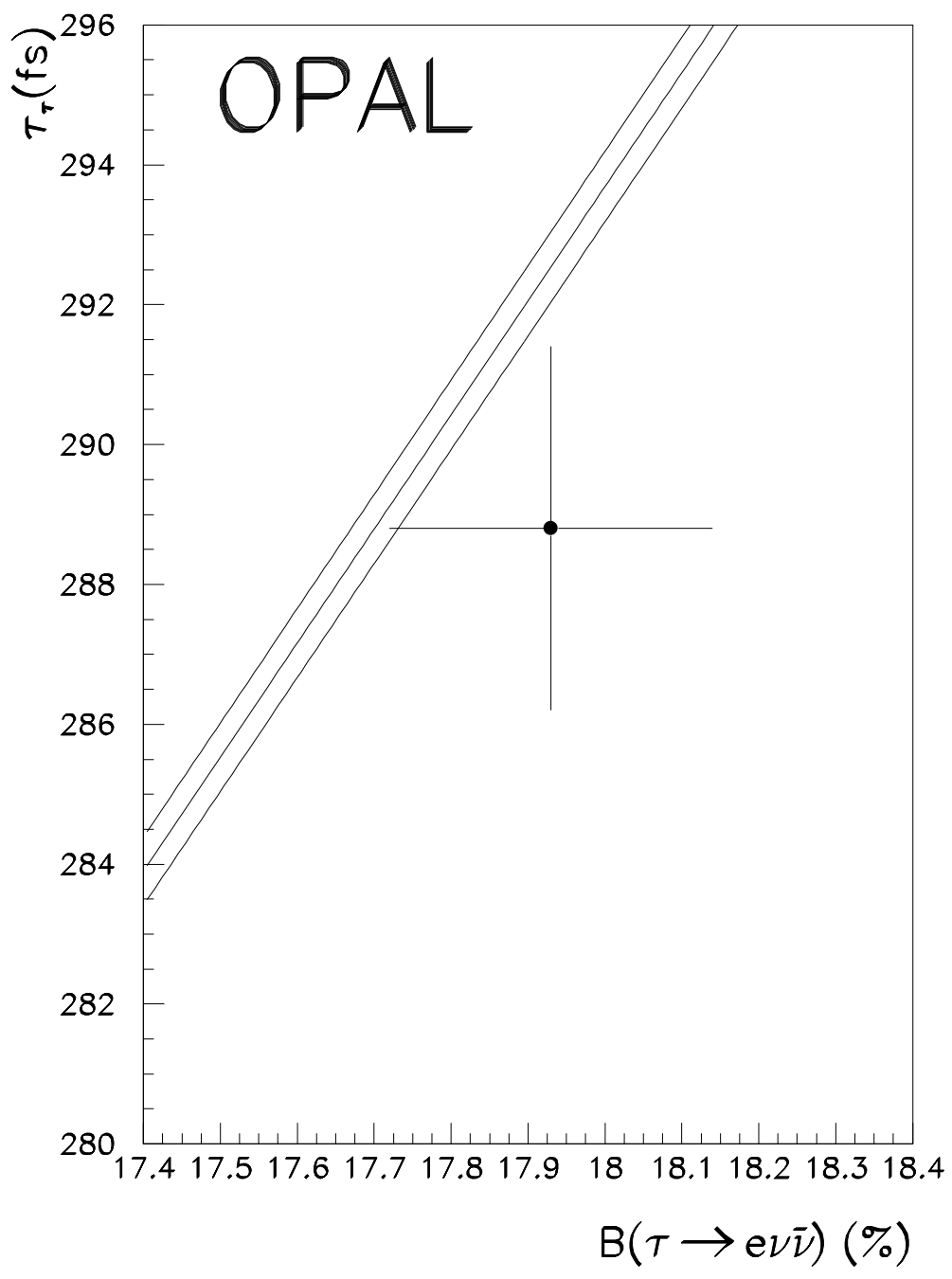

Figure 8: The average tau leptonic branching ratio described in this paper plotted against the OPAL lifetime meaurement [19]. The line displays the standard model relation between these quantities for a tau mass of $1777.1_{-0.5}^{+0.4} \mathrm{MeV}[14]$. 